\title{
1987 Calibration of the TFTR Neutron Spectrometers
}

\author{
Cris W. Barnes* and J. D. Strachan \\ Plasma Physics Laboratory, Princeton University \\ Princeton, N. 08543
}

\begin{abstract}
The ${ }^{3} \mathrm{He}$ neutron spectrometer used for measuring ion temperatures and the NE213 proton recoil spectromeier used for triton burnup measurements were absolutely calibrated with DT and DD neutron generators placed inside the TFTR vacuum vessel. The details of the detector response and calibration are presented. Comparisons are made to the neutron source strengths measured from other calibrated systems.
\end{abstract}

\section{DASCLAIMER}

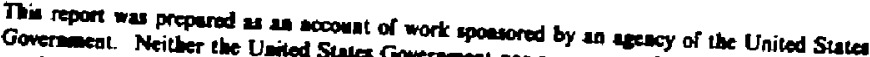

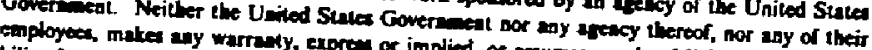
bility for the ecenrucy, completesen or or inplied, of anumes any lepal liability of reaposiproceses diectowed, or represents that its use would of any information, apparatus, product, or

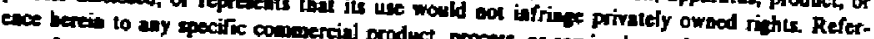

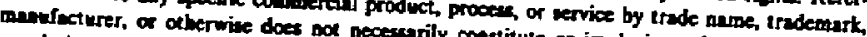

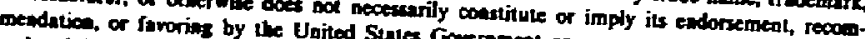
and opinions of authors expresed hereis do not reat or any afeacy thereof. The view

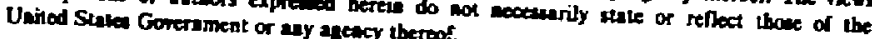

-Permanent Addres: Los Alumos National Laboratory, Los Alamos, NM 97545 


\section{Contents}

I Introduction $\quad \mathbf{3}$

2 Calibration Procedure 4

2.1 NE213 Proton Recoil Spectrometer ............... 5

$2.2{ }^{3}$ He Jonization Chamber ................... 11

2.3 Yeutron Generator and Operation ............... 13

3 Detector Response $\quad 18$

3.1 NE213: Rise-Time and Energy Spectra . . . . . . . . . . . . 18

$3.2{ }^{3}$ He Energy Spectra . . . . . . . . . . . . . . . . . . . 18

4 Collimator Apertuie Map and Detector Efficiencies 24

4.1 NE213 Detector . . . . . . . . . . . . . . . . . . . . 24

4.1.1 Aperture Maps .................... 24

4.1.2 Neutron efficiencies .................. 24

4.1.3 Comparison of yields to other calibrated systems ...... 30

$4.2{ }^{3} \mathrm{He}$ Detector ........................ 33

4.2 .1 Aperture map and scattering ............. 33

4.2 .2 Detector efficiency . . . . . . . . . . . . . . 37

4.2.3 Comparison to plasma yields .............. 38

5 Conclusions $\quad 39$

\section{List of Figures}

1 Plan of neutron collimator ................. 5

2 Photograph of neutron collimator ............... 6

3 Detailed view of Bay $F \ldots \ldots . \ldots \ldots$

4 The circuit diagram of the NE213 system. . . . . . . . . . . 8

5 VE213 rise-time spectra from PuBe source . . . . . . . . . . 10

6 NE213 energy-to-channel calibration . ........... 12

7 Diagram of the neutron generator ............... 14

8 Directed yield versus toroidal angle . . . . . . . . . . . . . 15

9 NE213 rise-time and energy spectra due to DD and DT generators . . 19

10 Overview of ${ }^{3}$ He energy spectra ................. 21 
$11{ }^{3} \mathrm{He}$ energy spectrum from Ohmic discharges compared to detector re-

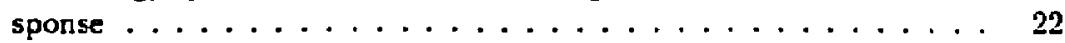

12 Details of the ${ }^{3} \mathrm{He}$ energy spectrum seen with generator . . . . . . 23

13 NE213 collimator aperture maps . . . . . . . . . . . 25

14 Rotation and vertical displacement data for DD generator . . . . 26

15 Calculations of collimator view of neutron profiles . . . . . . 27

16 Plasma yield cross comparison of NE213 and NE system . . . . . , 32

17 DT source strength comparison of NE213 and NE system . . . . . . 34

$18{ }^{3} \mathrm{He}$ DT aperture map from the recoil edge . . . . . . . 35

$19{ }^{3}$ He aperture maps from the thermal peak and DD recoil edge ... . 36

\section{List of Tables}

1 LOCUS database of calibration data $\ldots \ldots \ldots \ldots \ldots \ldots \ldots$

2 NE213 Background Rates . . . . . . . . . . . . . . 18

3 Intervening materials and their removal cross sections . . . . . . . 29

4 Database of DD yield comparisons . . . . . . . . . . . 31

$5{ }^{3} \mathrm{He}$ detector efficiency from $3 \mathbf{~ h r}$ generator run . . . . . . . . $3 i$

$6{ }^{3} \mathrm{He}$ Detector Efficiency (counts per neutron) for Plasma Neutrons .. 38

\section{Introduction}

This report documents the absolute calibration of the TFTR neutron spectrometers petformed in December 1987. The neutron spectrometers were located in a neutron collimator $15 \mathrm{~m}$ from the torus which viewed Bay $\mathrm{F}$ nearly perpendjcular to the plasma current. Two spectrometers were calibrated: (a) a ${ }^{3} \mathrm{He}$ ionization chamber ${ }^{1}$ which has been used $[1-3]$ for measurement of the Doppler broadening of the $d(d, n)^{3} \mathrm{He} 2.45-\mathrm{MeV}$ neutron emission; and (b) a NE213 proton recoil spectrometeri4] which has been used[5] for measurement of the magnitude of the $d(t . n) a$ 14-MeV neutron emission from the burnup of the $d(d, p) t 1-11 \mathrm{~V} V$ triton fusion product.

The calibration was accomplished by measuring the detector point efficiencies (counts per emitted neutron) across the collimator aperture separately with calibrated 2.5-MeV and 14-MeV neutron generators, and then integrating toroidally to simulate the respouse to a line source. The DT calibration of the NE213 spectrometer provides an absolute magnitude of the $14-\mathrm{MeV}$ neutron emission, and thus compliments the

\footnotetext{
'Model FNS-1 Seforad (Israel), purchased circa 1972.
} 
DT calibrations of the neutron activation systemi6], surface barrier detector: 7 ! and the ${ }^{238} U$ fission detectors [8] which also provide some measurements of the triton burnup. The NE213 DT calibration is more accurate than the other systems because the collimation provides a relatively more complete angular coverage and the high efficiency of the NE213 ensures good statistical accuracy. Obtaining the triton burnup from the ratio of $14-\mathrm{MeV}$ events to $2.5-\mathrm{MeV}$ events in the same detector also helps remove some systematic uncertainties in the burnup measurement, and was done to $20 \%$ accuracy. The DD calibration of the ${ }^{3} \mathrm{He}$ ionization chamber supplements the DD calibration of the fission chamber neutron detectors $[9,10]$, although the fission chambers (the XE system) are thought to be calibrated to higher precision. The DD calibration of the NE213 spectrometer was obtained with about $30 \%$ uncertainty, which is comparable to the precision of the fission detector calibration using the generator.

\section{Calibration Procedure}

The neutron collimator (Figure 1) contains $2{ }^{3} \mathrm{He}$ ionization chamber surrounded by a two-inch thick lead cylinder with a NE213 liquid scintillator placed immediately behind the lead cylinder. The collimatori11] is composed of $\mathrm{Li}_{2} \mathrm{CO}_{3}$ and paraffin mixed in equal volumes. It views the plasma through a double conical apertureil1! which is designed to minimize the flux of forward scatlered neutrons (from the aperture) onto the detectors. The collimator views TFTR Bay F (Figures 2 and 3 ) at a $3.6^{\circ}=0.1^{\circ}$ angle from the perpendicular, under the horizontal Pulse Height Analysis system vacuum tube. The collimator was aligned with respect to a laser beam that passed through the vessel minor axis in the center of Bay F, the center of both collimator cones, and the center of the detector casing. The laser was positioned just outside the vacuum vessel. The alignment uncertainty of $=10 \mathrm{~cm}$ was due to the blind projection of the beam into the ressel. From the dimensions of the edges of the cones of the collimator the optical cone angle of the collimator was $d=33 \pm 1 \mathrm{mRad}$, leading to an expected angle $\phi=360^{\circ} L d /\left(2 \pi R_{0}\right)=(10.2=0.3)^{\circ}$ of toroidal extent for a torus of major radius $R_{0}$ at a distance $L=14.7 \mathrm{~m}$ away. The geometrical factor of the XE213 spectrometer (the ratio of those unscattered neutrons passing through the detector to the total emitted) was expected to be

$$
g=\frac{A \phi}{4 \pi^{2} R_{0} L}=3.7 \times 10^{-\mathrm{s}}
$$

for an extended line source of toroidal radius $R_{0}=2.7 \mathrm{~m}$ and a detector area $A=$ $20.3 \mathrm{~cm}^{2}$, and no attenuation. 


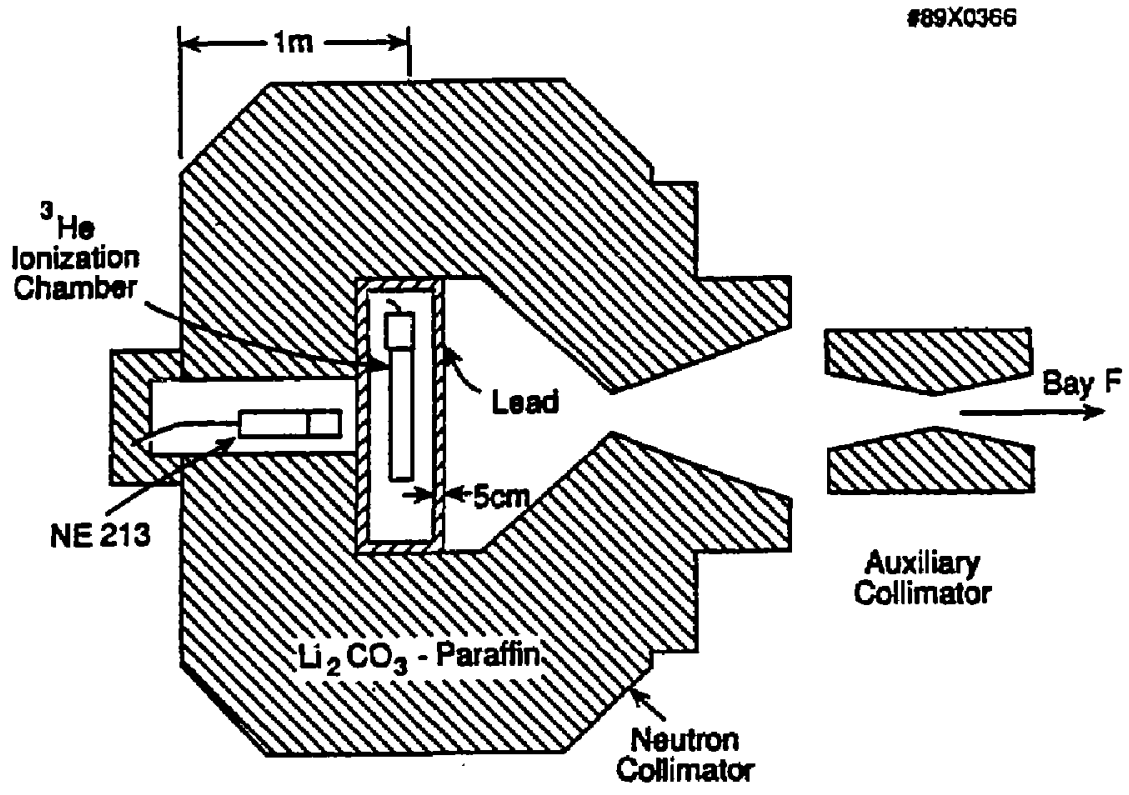

Figure 1: Plan of neutron collimator with double conical aperture, lead shield, ${ }^{3} \mathrm{He}$ iunization chamber and NE213 liquid scintillator.

\subsection{VE213 Proton Recoil Spectrometer}

The detector consisted of a 2 inch high by 2 inch diameter XE213 liquid scintillator (detector area $A=20.3 \mathrm{~cm}^{2}$ ), encased in a reflecting $c y$ rlinder with a transparent window at one end. An RCA885T photomultiplier tube (PMT) was attached to the window and had a RCA2209.A P.IT base.

The circuit used is shown in Figure 4 . The PMT was run at $-1600 \backslash$ from an Ortec $4 \overline{5} 6$ high roltage supply. The anode output was run through $R G / 6293 \Omega$ cable to the input of an Ortec 474 Timing Filter Amplifier set with 20 nsec integration and 20 nsec differentiation at $\times 10$ gain. That output was put into an Ortec $436100 \mathrm{MHz}$ Discriminator.

There are two parallel circuits after the discriminator. each centered at different energies. with one around 2.5.MeV neutron energy and one around $1 \mathrm{H}$. Mel. Each of the two discriminator outputs were put into a Ortec $416 \mathrm{~A}$ Gate and Delay Generator. and the Delayed Marker output was used for the Stop signal in an Ortec 467 Timeto-Pulse Height Converter (TPHC). The dynode output of the PMT base was sent through RG/58L $51 \Omega$ cable to an Ortec 452 Spectroscopy. Amplifier set a: about $\times 40$ 


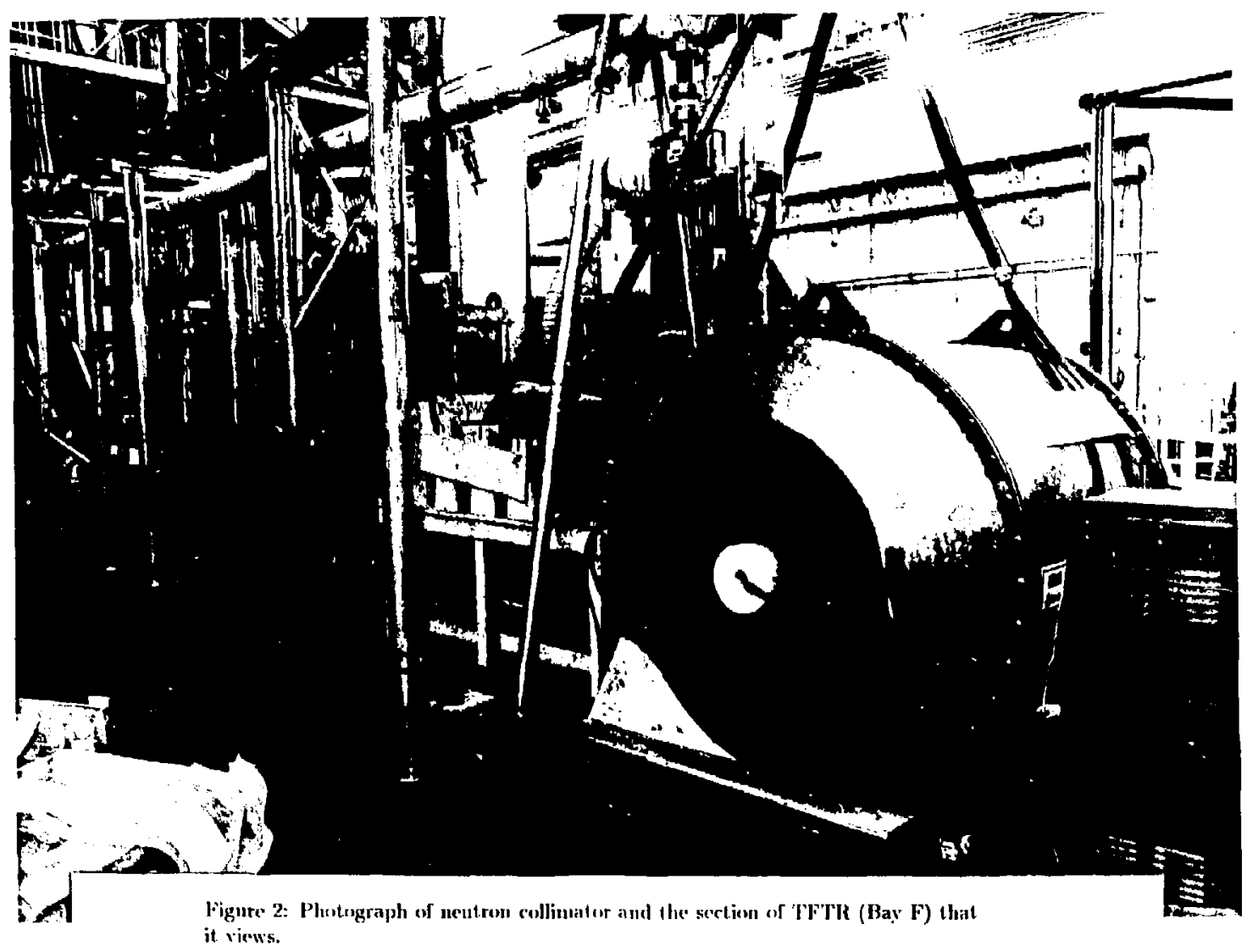

Fignte 2: Plontugraph of neutron collintater and the section of TFTR (Ray F) that it riews. 

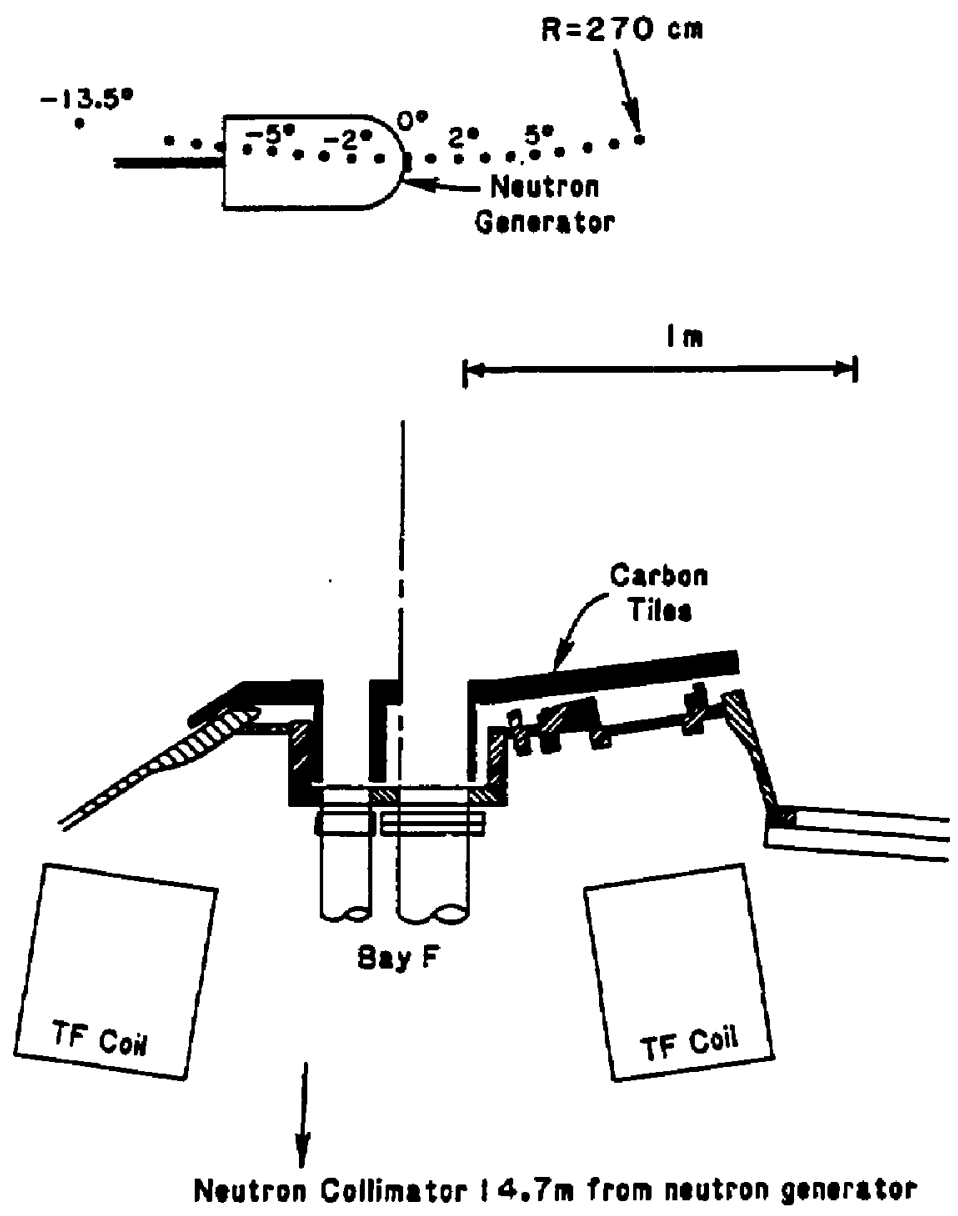

Figure 3: Plan view of the TFTR Bay F area. The major components are the toroidal magnetic field coils, the stainless steel vacuum vessel and flanges (hatched regions). and the carbon protective plates (black regions) which are supported by a $3 / 8$ inch Inconel backing plate. 


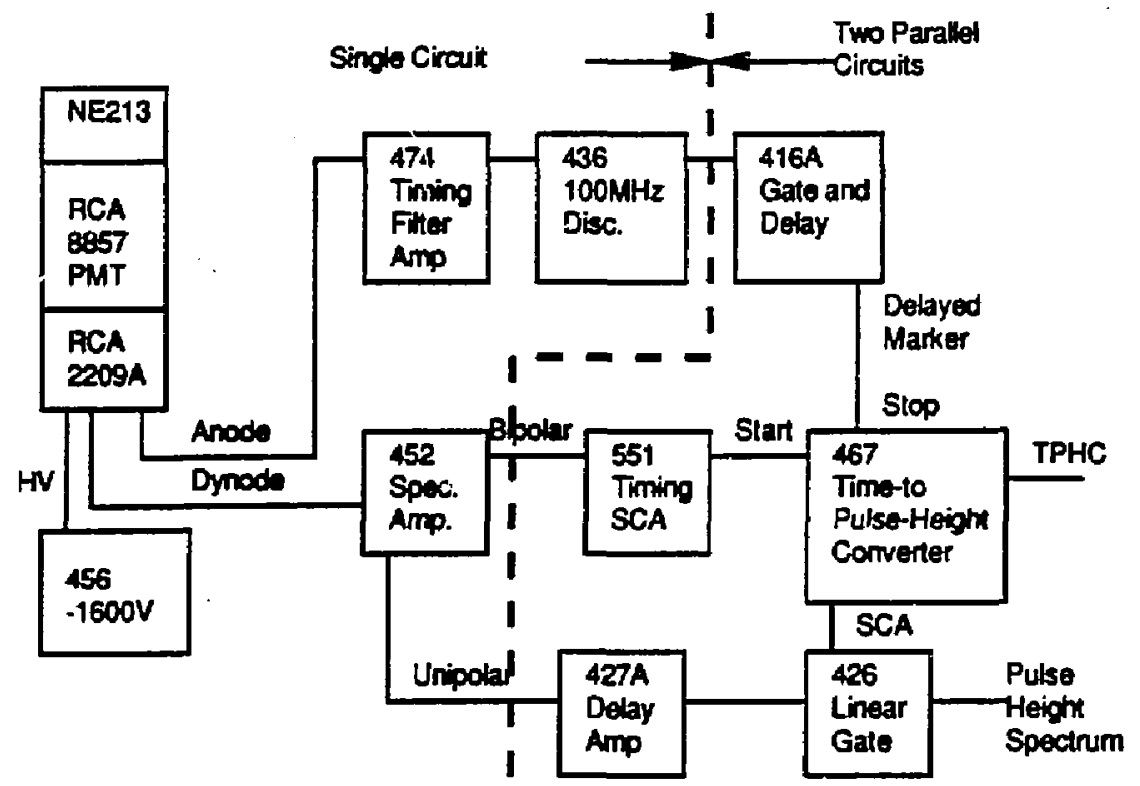

Figure 4: The circuit diagram of the XE213 system.

gain. with a $0.25 \mu \mathrm{sec}$ shaping constant and a built in $2 \mu \mathrm{sec}$ delay on the sut put. The Bipolar outpur was split into two parallel circuits. each of which goes into separate Ortec 551 Tizring Single Channel Analyzers (TSCA). The Negative output of the TSCA was used as the Start of the TPHC. L'sing the slow dynode signal for the Start reduces the number of triggers of the TPHC for out-of-range pulse heights. and causes the neutron rise-times to be displayed before the ? rise timesil2].

The C'nipolar output of the amplifier was split. and each signal sent through a 427A Delay Amplifier to a 426 Linear Gate. The SCA output of the TPHC was then used as the Enable of the Gate. with the output sent to histogrammers of the TFTR data acquisition system (CIC ADA). The TPHC output was also sent to a histogrammer.

The pulse-shape discrimination circuit was set up by:

1. Using a ${ }^{137} \mathrm{C}$ 's $\%$ ray source $(0.66-\mathrm{Mel})$. the discriminator of the 436 unit was set on the Compton recoil edge (at a value above which the discriminator output sate would sharply reduce to near zero). 
2. The Lower Level Discriminator (LLD) on the 551 was set to not further discriminate against the ${ }^{1: 17} \mathrm{Cs}$ pulses.

3. Using a ${ }^{22} \mathrm{Na} \gamma$-ray source (1.25 MeV), the Upper Level Discriminator of the j5l was set just above the Compton recoil edige (below which the output would sharply increase from near zero).

4. The TPHC pulses from the 467 unit were made to be several volts by varying the Delay of the 551. The Timing Walk of the 551 was varied until the most narrow band of rise-time pulses was obtained.

5. A PuBe neutron- $\gamma$ source was used to confirm that the neutron rise-time pulses were separated and below the $\gamma$-ray pulaes.

6. Energy spectra with the available $\gamma$-sources $\left({ }^{137} \mathrm{Cs},{ }^{65} \mathrm{Zn},{ }^{22} \mathrm{Na}\right.$, and ${ }^{85} \mathrm{Y}$ were used in the calibration) were used to determine an energy calibration from the Compton edges.

7. The ULD and the LLD of the SCA on the TPHC were set on either side of the neutron peak.

8. For the $14 \mathrm{MeV}$ branch of the circuit, the LLD of the TSCA was set just below the Compton recoil edge of the $4.43 \mathrm{MeV} \gamma$ from the PuBe source. This edge was hard to see and the setting not very accurate (channel 60 instead of 90). The setting of the ULD was insensitive since it can be set quite high without a dynamic range froblem.

Unfortunately, the TPHC unit drifts slightly day-to-day, making the rise-time spectrum shift channels by $1-5 \%$. This drift can easily allow photon pulses to be gated into the energy spectrum. However, unique identification of neutron events is possible by using the rise time spectrum itself and just integrating under the neutron peak. The rise-time spectra obtained from the PuBe source (Figure 5) have a "figureof-merit" (peak separation divided by the sum of the FWHM of the two peaks) of 1.8 or better. The width of the rise-time peaks and their separation have been observed to be constant to within $1 \%$ between spectra taken at different times below neutron source strengths of $3 \times 10^{14} \mathrm{sec}^{-1}$. The neution counts are found by fitting the $\gamma$ peak with a Gaussian, moring 3 standard deviations below the peak of the $\gamma$ 's (typically 26 channels for the low energy circuit and 19 channels for the high energy circuit), and summing down for 80 channels for the low energy circuit and 65 channels for the high (equivalent to \pm 2 standard deviations of the neutron rise-time peak). Thus, a $14-\mathrm{MleV}$ rate and combined $2.5-\mathrm{MeV}$ and $14-\mathrm{MeV}$ rate are available. 


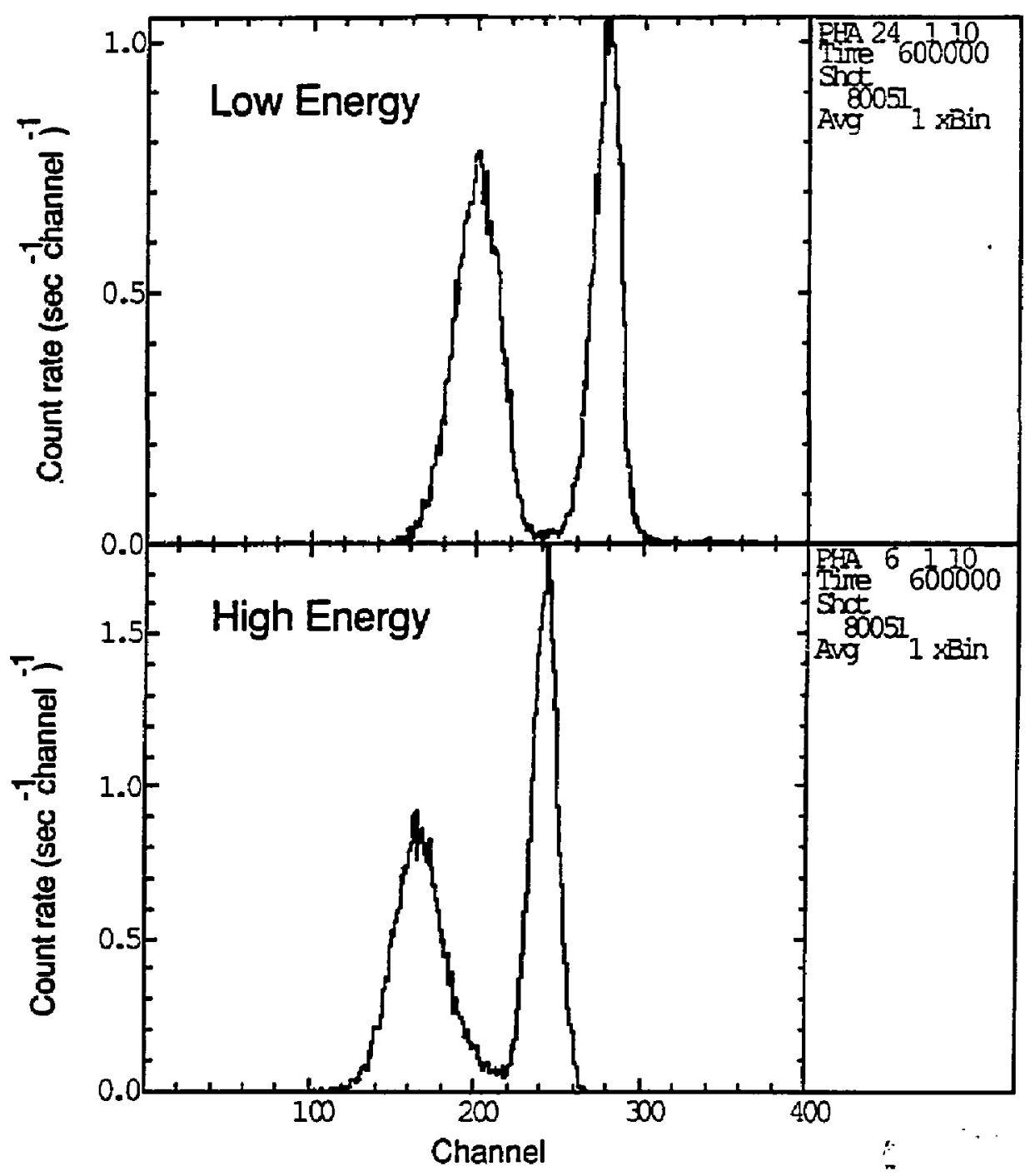

Figure 5: Pulse-shape discrimination rise-time spectra from the two parallel circuits using a PuBe neutron- $y$ source. 
In any pulse-shape discrimination circuit the magnitude of the contribution to the neutron counts from $\gamma$ 's must be considered. For a pure $\gamma$ source (such as ${ }^{22} \mathrm{Na}$ ) the fraction of gamma counts observed inside the lower energy neutron windew is about 1 in $\mathbf{5 0}$. Estimating the $\gamma$ events under the neutron peal in a timing spectrum taken with a neution source is more difficult. The rise-time spectrum of the $\gamma$ 's may not be Gaussian, but may have exponential tails. Assuming a worse case of exponential tails the $\gamma$-rejection (fraction of counts under the neutron peak due to $\gamma$ 's) was still better than 1 part in $\mathbf{5 0}$ in the our energy channel. When the algorithm described above of finding and summing under the neutron peak is used, the resulting neutron rates are expected to have $95 \%$ of all neutron counts and less than $2 \%$ of any gamme counts. With a typical $\gamma$-to-neutron ratio of 3 or iess at the detector, these $\mathrm{DD}$ rates have a relative uncertainty in comparing discharges of less than $8 \%$ from this method of analysis. The $\gamma$-rejection improves significantly at higher energy, and hence is over 100 for the high energy channel. The rejection cannot be measured directly because a $\gamma$ source was not avajable above the biss setting used. But in plasma discharges less than 1 in 100 of the $\gamma$ 's appear in the neutron channel.

The energy caljbration of the spectrometer is shown in Figure 6. The channels in the energy spectrum where the Compton recoil edges appear for various $\gamma$ sources[16,14], along with the channels of the DD and DT neutron recoil edges, determine the channel-to-energy calibration. This is only important as a diagnostic check, and in comparing the expected physical efficieusy (from the detector counting efficiency times the geometrical factor) with the absolute calibration.

The discriminator settings, combined with the energy calibration, allow determinatien of the counting efficiency of the two channels. Integrating over the data of Verbinskif 13 ] an absolute counting efficiency of the NE213 for 14-MeV neutrons is obtained of $(5.7 \pm 0.2) \times 10^{-2}$. The absolute counting efficiency for $2.5-\mathrm{MeV}$ neutrons is about $(3.0 \pm 1.0) \times 10^{-2}$, where the large error is because the bias is set very close to the $2.5 \mathrm{MeV}$ recoil edge and hence the counting efficiency is very sensitive to small changes in the bias. The absolute counting efficiency for 14-MeV neutrons in the lower energy window is $(1.8 \pm 0.2) \times 10^{-2}$. The ratio of counting response in the two circuits to $14-\mathrm{MeV}$ neutrons is predicted to result in approximately $0.31 \pm 0.04$ counts in the lower energy window for every count in the higher energy window.

\section{2. ${ }^{3}$ He Ionization Chamber}

The ${ }^{3} \mathrm{He}$ ionization chamber was operated as in Refs. 2 and 3 with -2500 volts applied to it and a $12 \mu \mathrm{sec}$ shaping constant used on the main amplifier. 


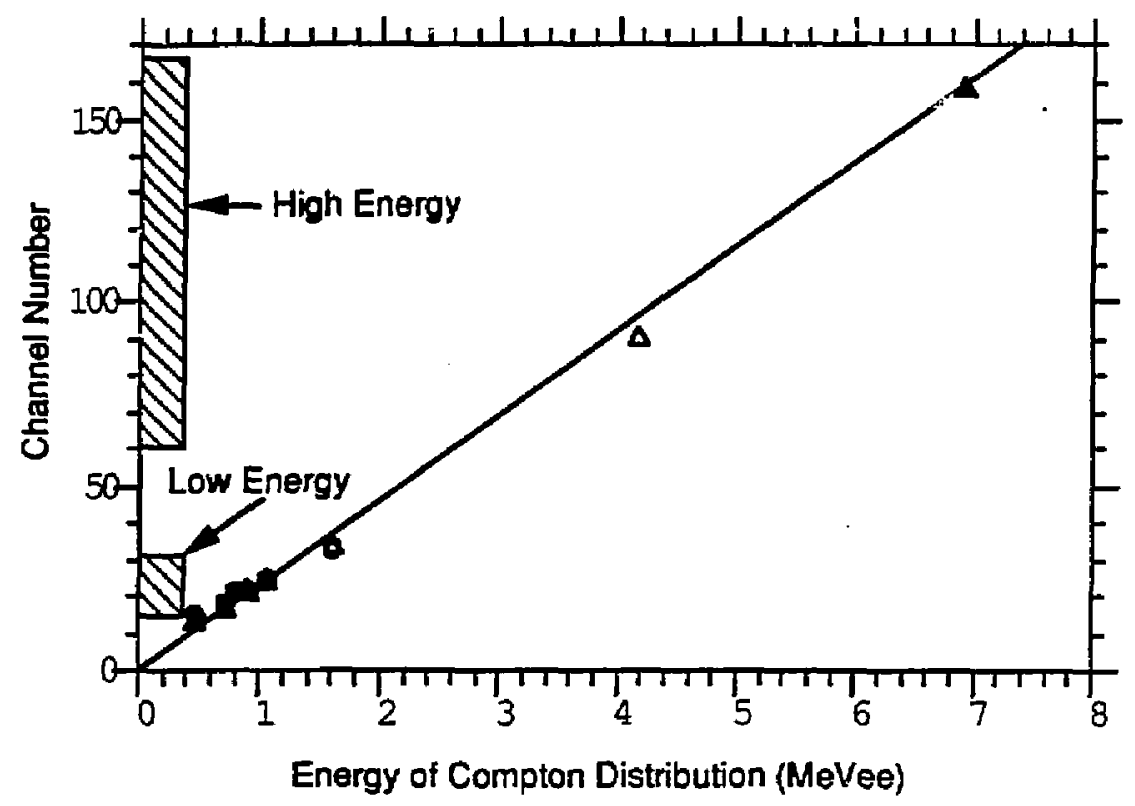

Figure 6: NE213 pulse-height energy calibration. The channel is plotted versus the energs of the Compton edge of the source $\left(2 E_{v}^{2} j\left(m_{e} c^{2}-2 E_{v}\right)\right)$. The circle and triangles are for the two different circuits. "Pulse Height Light Output" unitsi 13 ! are about $5 \%$ greater 14.15 , than using the half-height of the 22 . Ta edge (1.062 Meliee Mel electron equivalent) as one unit, and hence (within the accuracy of this energiv calibration) PHLO units are equivalent to the uruts of the ordinate. The observed locations of the DD and DT recoil edges are included in the graph with closed symbols. while the hatched regions show the channel ranges of the parallel energy circuits isee Figs. $9(\mathrm{~b})$ and $9(d)$. 


\subsection{Neutron Generator and Operation}

A neutron generator ${ }^{2}$ (DD emission level of $2 \times 10^{8} \mathrm{sec}^{-1}$ or DT emission level of $2 \times 10^{10} \mathrm{sec}^{-1}$ ) was placed inside the vessel and pointed along the plasma minor axis (Fig. 3) in a number of locations which scanned TFTR Bays F and G. The neutron emission from the generator was measured in the forward direction by a ${ }^{238} U$ fission detector, and the fission detector was calibrated by activation foils[6] placed (Figure 7) immediately in the forward direction (Indium for the DD generator head and Aluminum for the DT generator head). The fluence through the foils was determined by the activation to about $12 \%$ uncertainty, including about $2 \%$ from statistics, $8 \%$ from uncertainty in the counting efficiency of the $H P G e$ counting system, $4 \%$ in the generator target-foil distance (1 $\mathrm{mm}$ ), and $5 \%$ in the energy-dependent cross-section. (Cross-sections of 347 mbam for Indium at $2.76 \mathrm{MeV}$ 'forward emission energy) and 111 mbarn for aluminum at $14.55 \mathrm{MeV}$ were used.)

The angular emission of the generator has been previously measured[17] using an NE213 spectrometer. No data exists on the azimuthal symmetry of the emission, and in this paper it has been assumed to be uniform. (Note the collimator sightline was 12 degrees low.) From the data of Ref. 17, the total neutron emission in all directions relative to the emission in the forward direction was $0.56 \times 4 \pi$ for the DD generator and $0.76 \times 4 \pi$ for the DT generator with $8 \%$ uncertainty from model dependent choices of how to integrate the angular data and deal with missing data at some angles, and on how to normalize to the $0^{\circ}$ emirsion. The total "yield" of the generator on each shot is the monitor fission chamber counts divided by its calibrated efficiency for total geutron emission. The efficiencies are $2.83 \times 10^{-7}$ for DD and $5.25 \times 10^{-7}$ for DT, with $15 \%$ overall uncertainty.

The collimated detectors used here primarily see unscattered, full-energy neutrons, or in other words they see the generator emission in the direction of the detectors. That "directed yield" is the total yield times the relative emission at the angle of observation; that is, it is the yield as if the generator emitted isotropically at the same magnitude that it emits in the direction of the detector. The "point efficiency" is the ratio of the detector counts to the directed yield. The directed yield towards the neutron collimator varied by about $20 \%$ for the positions of the generator used in the calibration (Figure 8).

For each experiment or "shot," the TFTR data acquisition computer was triggered, and then the generator was turned on, run for the necessary duration, and then turned off. The total integrated counts in the monitoring detector and the detectors being calibrated were then tabulated and analyzed. The gain of the monitor detector electronics drifted $<1 \%$ shot-to-shot, but with occasional overnight changes of up

\footnotetext{
${ }^{2}$ Karman Model A71I neutron generator with either $\triangle$ DD or a DT head.
} 
\# 88X0875

\section{GEOMETRY OF NEUTRON GENERATOR}
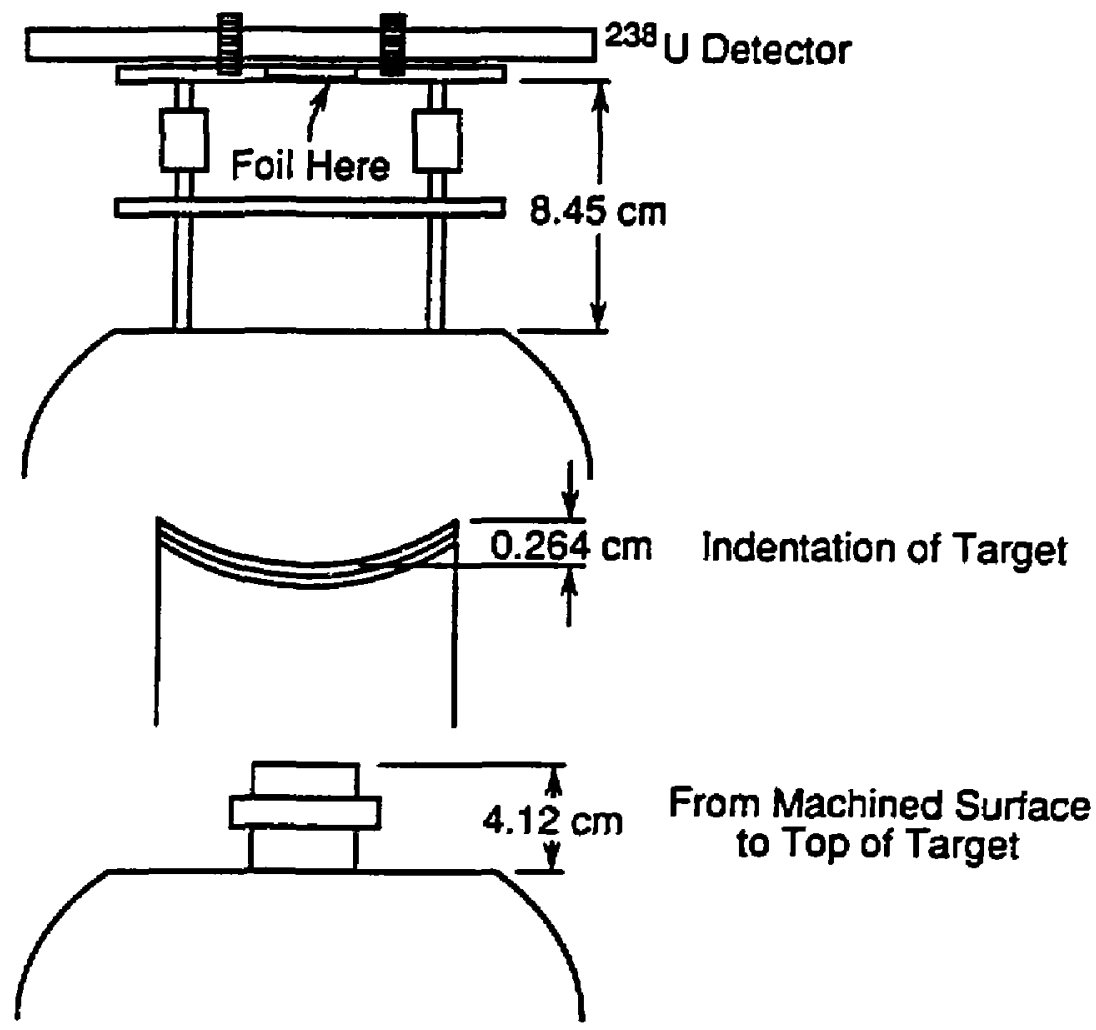

Total Distance: +8.45

$-4.124 .59 \mathrm{~cm} \pm 0.10 \mathrm{~cm}$

Figure $i$ : Diagram of the neutron generator showing location of ine activation foils and the monitor fission chamber used. 


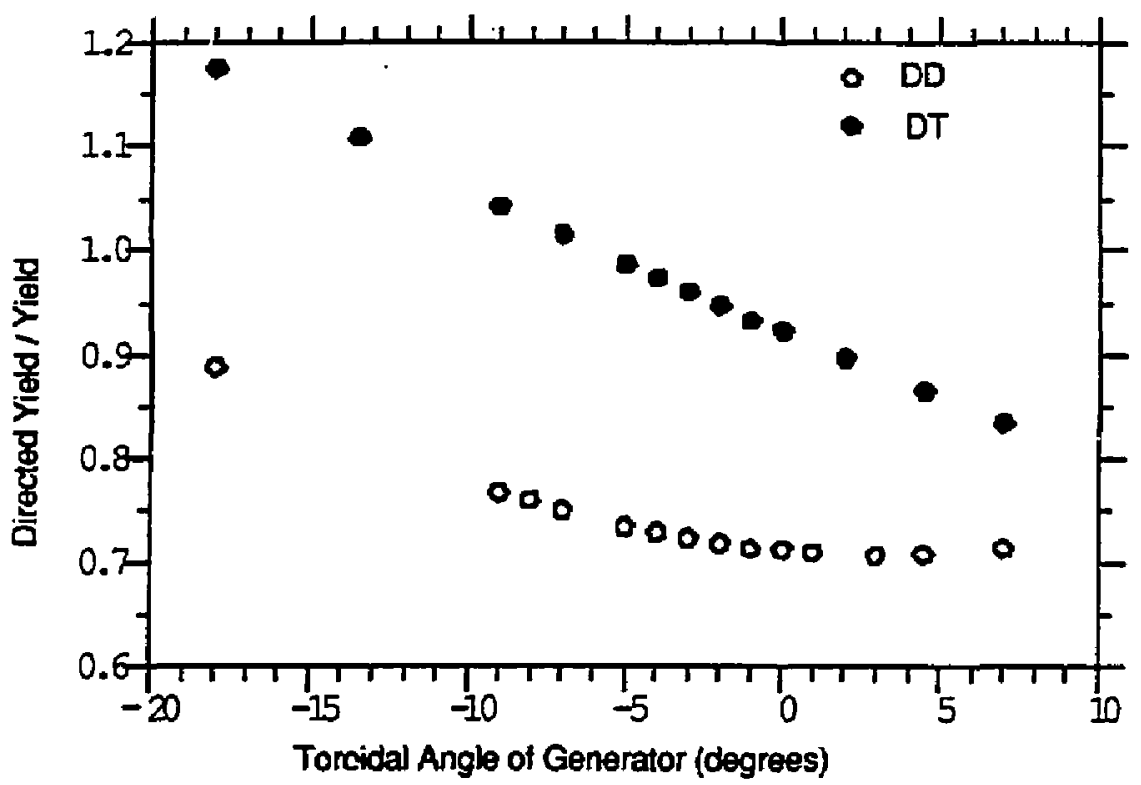

Figure 8: Ratio of generator directed yield to total yield versus toroidal angle of the generator. The squares are for DT and the circles for DD. The ratio has been calculated at each generator position from the data of Ref. 17 .

to $4 \%$. This was observed from variations in the peak of a calibrated pulser. The channel of the peak of the fission spectrum stayed proportional to the channel of the pulser (at $5 \bar{\tau}=1 \%$ ). The monitor detector count was taken as the sum of the counts in the fission spectrum between $34.8 \%$ and $91.3 \%$ of the pulser channel for each shot. Lncertainties of $<1 \%$ arise from 1 channel uncertainties in the spectrum integration and counting statistics.

The calibration consisted of

1. a scan of 10 minute $D D$ generator runs in the Bay $F$ area.

2. a single $3 \mathrm{hr} \mathrm{DD}$ generator run at one location near the maximum sensitivity derived from the Bay $F$ scan. and

3. a scan of two minute DT generator runs in the Bay $F$ area.

(There was additional uork done for detectors at Bay M.)

The cables for the neutron generator entered the TFTR vacuum vessel through an opening at Bay $\mathrm{K}$. and the generator was always pointed away from that Bay. 
Hence, for the work near Bay $F$, the genetator was always pointing counter-clockwise when viewed from above. The head of the generator was located on its cart above marks positioned on a work floor, with the toroidal angle measured from the center of Bay $F$ in a counter-clockwise from above direction (so that the toroidal angle increases from left to right when viewed from the collimator). The target of the generator was positioned at $270 \mathrm{~cm}$ major radius, with the beam tangential to the torus at that radius. Two data points were taken at $280 \mathrm{~cm}$ major radius, and a single data point was taken with the DD generator beam pointed outward by $45^{\circ}$. All but two shots were taken at a $z$-position of 0 in the center of the vacuum vessel; those two shots had the DD generator raised by 15 or $30 \mathrm{~cm}$. The generator was moved to different torojdal angles for each data acquisition "shot."

Table 1 is a printout of the data from all the Bay F calibration shots. 


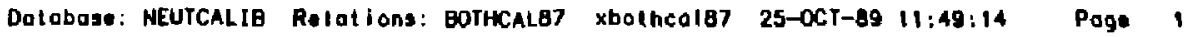

SHOT NSORT AMGLE RQ ZGEN DIR TGTIME MONINT YIELO OIRYIELD DIST VIEW DD213 CORDD OT213 CORDT

\begin{tabular}{|c|c|c|c|}
\hline $\begin{array}{l}90016 \\
90017 \\
90020 \\
90021 \\
90822 \\
90023 \\
90024 \\
90025 \\
90026 \\
90027\end{array}$ & $\begin{array}{l}1 \\
1 \\
1 \\
1 \\
1 \\
1 \\
1 \\
1 \\
1\end{array}$ & $\begin{array}{r}0.0 \\
-4.5 \\
5.0 \\
9.0 \\
18.0 \\
7.0 \\
3.0 \\
1.0 \\
-1.0 \\
-3.0\end{array}$ & $\begin{array}{l}270 \\
279 \\
270 \\
270 \\
278 \\
270 \\
270 \\
270 \\
270 \\
270\end{array}$ \\
\hline $\begin{array}{l}90028 \\
90029 \\
90031 \\
90031 \\
90032 \\
90033 \\
90034 \\
90035 \\
90036 \\
90037\end{array}$ & $\begin{array}{l}1 \\
1 \\
1 \\
1 \\
1 \\
1 \\
1 \\
1 \\
1 \\
1\end{array}$ & $\begin{array}{r}-7.0 \\
2.0 \\
4.0 \\
0.0 \\
2.0 \\
2.0 \\
3.0 \\
2.0 \\
2.0 \\
2.0\end{array}$ & $\begin{array}{l}270 \\
270 \\
270 \\
270 \\
270 \\
270 \\
270 \\
270 \\
270 \\
270\end{array}$ \\
\hline $\begin{array}{l}90038 \\
90039 \\
90040 \\
90041 \\
90042 \\
90071 \\
90072 \\
90073 \\
90074 \\
90075\end{array}$ & $\begin{array}{l}1 \\
1 \\
1 \\
1 \\
1 \\
2 \\
2 \\
2 \\
2 \\
2\end{array}$ & $\begin{array}{l}2.0 \\
2.0 \\
2.0 \\
2.0 \\
2.0 \\
2.0 \\
3.0 \\
4.0 \\
7.0 \\
9.0\end{array}$ & $\begin{array}{l}270 \\
270 \\
270 \\
270 \\
270 \\
270 \\
270 \\
270 \\
270 \\
270\end{array}$ \\
\hline $\begin{array}{l}90077 \\
90079 \\
96080 \\
90083 \\
90084 \\
90085 \\
90086 \\
96089 \\
90090 \\
90091\end{array}$ & $\begin{array}{l}2 \\
2 \\
2 \\
2 \\
2 \\
2 \\
2 \\
2 \\
2 \\
2\end{array}$ & $\begin{array}{r}1.0 \\
0.0 \\
-7.0 \\
-4.5 \\
-4.5 \\
-4.5 \\
16.8 \\
13.5 \\
5.6 \\
3.0\end{array}$ & $\begin{array}{l}270 \\
270 \\
270 \\
270 \\
270 \\
270 \\
270 \\
270 \\
270 \\
270\end{array}$ \\
\hline $\begin{array}{l}90092 \\
90093 \\
90094 \\
90095 \\
96096 \\
90097 \\
90098\end{array}$ & $\begin{array}{l}2 \\
2 \\
2 \\
2 \\
2 \\
2 \\
2\end{array}$ & $\begin{array}{l}-2.0 \\
-4.5 \\
-4.5 \\
-4.5 \\
-4.5 \\
-4.5 \\
-4.5\end{array}$ & $\begin{array}{l}270 \\
280 \\
280 \\
280 \\
280 \\
280 \\
280\end{array}$ \\
\hline
\end{tabular}

$\begin{array}{llllll}660 & 4.844 E+04 & 1.712 E+11 & 1.218 E+11 & 1467 & 93.5 \\ 660 & 4.984 E+04 & 1.761 E+11 & 1.247 E+11 & 1469 & 96.8 \\ 660 & 5.014 E+04 & 1.772 E+11 & 1.299 E+11 & 1466 & 67.7 \\ 660 & 5.044 E+04 & 1.782 E+11 & 1.366 E+11 & 1468 & 63.0 \\ 660 & 4.862 E+04 & 1.718 E+11 & 1.524 E+11 & 1477 & 72.5 \\ 660 & 1.846 E+04 & 1.712 E+11 & 1.262 E+11 & 1467 & 85.3 \\ 660 & 5.009 E+04 & 1.798 E+11 & 1.299 E+11 & 1466 & 90.0 \\ 660 & 5.026 E+04 & 1.774 E+11 & 1.265 E+11 & 1466 & 92.5 \\ 660 & 5.029 E+04 & 1.777 E+11 & 1.268 E+11 & 1467 & 94.7 \\ 660 & 5.185 E+04 & 1.032 E+11 & 1.296 E+11 & 1468 & 97.0\end{array}$

$660 \quad 4.826 E+04 \quad 1.705 E+11 \quad 1.215 E+111471181.7$

$6605.140 E+64 \quad 1.816 E+11 \quad 1.393 E+111466 \quad 91.2$

$6685.172 E+641.820 E+11 \quad 1.330 E+111466$ Ba.8

$6605.156 E+441.822 E+11 \quad 1.361 E+11146804.1$

$6605.920 E+442.095 E+11 \quad 1.53 \mathrm{EE}+11$ 1456 01.2

668 5.035E+ $2.133 E+11 \quad 1.330 E+11 \quad 1466 \quad 91.2$

560 5.905E+04 2.115E+11 2.911E+11 1466 46.9

$1360 \quad 1.436 E+05$ 5.074E+11 $3.641 E+11$ 1460 01.2

1360 1.442E+05 5.095E+11 $3.656 E+11$ 1466 91.2

$1560 \quad 1.469 E+05 \quad 5.191 E+11 \quad 3.725 E+11 \quad 1466 \quad 91.2$

$1560 \quad 1.460 \mathrm{E}+05 \quad 5.150 \mathrm{E}+11 \quad 3.702 \mathrm{E}+11 \quad 146691.2$

$1560 \quad 1.476 E+55.216 E+11 \quad 3.742 E+11 \quad 146691.2$

$\begin{array}{rlllll}760 & 7.202 E+84 & 2.545 E+11 & 1.025 E+11 & 1466 & 91.2 \\ 1260 & 1.150 E+05 & 4.092 E+11 & 2.036 E+11 & 1466 & 91.2\end{array}$

$165 \quad 1.480 E+06 \quad 2.834 E+12 \quad 2.694 E+12 \quad 146891.2$

$150 \quad 1.580 E+06 \quad 3.025 E+12 \quad 2.005 E+12 \quad 1466 \quad 90.9$

150 1.314E+ $2.303 E+12 \quad 2.438 E+12 \quad 1466 \quad 88$.

$150 \quad 1.352 E+06 \quad 2.375 E+12 \quad 2.614 E+12 \quad 1467 \quad 05.3$

$1651.263 \mathrm{E}+06 \quad 2.406 \mathrm{E}+12 \quad 2.500 \mathrm{E}+12$ 1468

$150 \quad 1.133 E+\theta 6 \quad 2.158 E+12 \quad 2.012 E+12 \quad 1466 \quad \$ 2.5$

$150 \quad 0.368 E+65 \quad 1.784 E+12 \quad 1.646 E+12 \quad 1467 \quad 03.5$

$1659.473 E+05 \quad 1.004 E+12 \quad 1.505 E+121471101.7$

$903.851 E+65 \quad 7.335 E+11 \quad 6.343 E+11146998.8$

480 1. $067 E+66 \quad 3.061 E+12 \quad 2.047 E+12146990.8$

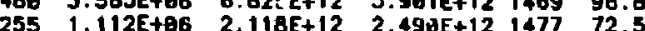

$\begin{array}{llllll}210 & 03 E+66 & 2.118 E+12 & 2.490 E+12 & 1477 & 72.5\end{array}$

180 7.31BE+65 $1.394 E+12$ 1.375E+12 1468 a7.7

$240 \quad 5.638 E+05 \quad 1.264 E+12 \quad 1.214 E+12146690.0$

$210 \quad 7.069 E+05 \quad 1.335 E+12 \quad 1.197 E+12 \quad 1468 \quad 95.9$

$420 \quad 5.734 E+05 \quad 1.092 E+12 \quad 9.444 E+11146998.8$

$150 \quad 1.126 E+\theta 5 \quad 7.859 E+11 \quad 6.796 E+11 \quad 146998.8$

$\begin{array}{lllllll}195 & 7.228 E+05 & 1.377 E+12 & 1.190 E+12 & 1469 & 98.8\end{array}$

$1867.586 E+85 \quad 1.445 E+12 \quad 1.249 E+12 \quad 1469 \quad 98.0$

$210 \quad 7.671 E+05 \quad 1.499 E+12 \quad 1.296 E+12 \quad 1469 \quad 98.8$

$2107.640 E+05 \quad 1.455 \mathrm{E}+12 \quad 1.258 E+12 \quad 1469 \quad 98.8$
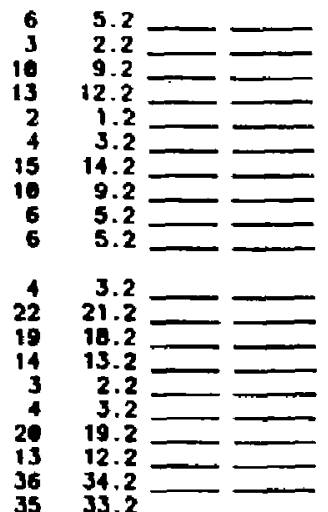

35.

$37 \quad 35.2$

$14 \quad 13.1$

464 463.8 $\overline{1205} \overline{1204.6}$

$516 \quad 515.8 \quad 1496 \quad 1495.7$

$477 \quad 476.8 \quad 1303 \quad 1362.7$

$177 \quad 176.8 \quad 345 \quad 344.7$

377376.810241823 .6

$\begin{array}{llll}265 & 264.0 & 691 & 690.7\end{array}$

$200 \quad 199.8 \quad 550 \quad 549.7$

$\begin{array}{llll}86 & 05.8 & 213 & 212.6\end{array}$

$218 \quad 59.9 \quad 161 \quad 160.8$

$\begin{array}{rrrr}376 & 575.5 & 1607 & 1605.9\end{array}$

$\begin{array}{llll}55 & 54.7 & 129 & 128.4\end{array}$

$\begin{array}{llll}29 & 28.8 \quad 73 & 72.5\end{array}$

$\begin{array}{llll}153 & 152.6 & 395 & 394.6 \\ 199 & 198.7 & 530 & 529.5\end{array}$

$\begin{array}{rrrr}54 & 53.8 & 155 & 154.5 \\ 77 & 76.5 & 243 & 242.0 \\ 76 & 75.8 & 177 & 176.7 \\ 127 & 126.8 & 312 & 311.5 \\ 119 & 118.8 & 358 & 357.6 \\ 138 & 129.8 & 369 & 368.5 \\ 134 & 133.8 & 358 & 357.5\end{array}$


Table 2: NE213 Background Rates

\begin{tabular}{c|ccccc} 
Detector & $\begin{array}{c}\text { Time } \\
\text { (secs) }\end{array}$ & $\begin{array}{c}\text { Neutron } \\
\text { counts }\end{array}$ & $\begin{array}{c}\text { n-Rate } \\
\text { (cps) }\end{array}$ & $\begin{array}{c}\gamma \\
\text { counts }\end{array}$ & $\begin{array}{c}\gamma \text {-rate } \\
\text { (cps) }\end{array}$ \\
\hline NE213 (DD) & 8700 & 10 & $1.15 \times 10^{-3}$ & 420 & $4.83 \times 10^{-2}$ \\
NE213 (DT) & 8700 & 20 & $2.30 \times 10^{-3}$ & 537 & $6.17 \times 10^{-2}$
\end{tabular}

\section{Detector Response}

\section{1. .VE213: Rise-Time and Energy- Spectra}

The rise-time spectra from the NE213 detector (Figure 9) to (a) the DD generator, and (c) the DT generator have significant pulse-shape discrimination. Using the algorithm described before and integrating between the channels as marked rejects less than $5 \%$ of the neutrons and accepts less than $2 \%$ of the gammas.

The energy spectrum for the DD generator [Fig. 9(b)] shows the recoil edge for the 2.5-MeV neutrons but no $14-\mathrm{MeV}$ neutrons. For the DT generator spectrum [Fig. 9(d)] the recoil edge at $14 \mathrm{MeV}$ is clearly seen. The enhanced counts at low energy are consistent with the expected detector response to $14-\mathrm{MeV}$ neutrons[13]. The dip seen in the higher channels is not consistent with the expected neutron response. However, for the DT data the TPHC had drifted significantly [the gamma peak had moved from channel 359 during the DD calibration to channel 340 as seen in Fig. $9(c)]$, so that $\gamma$ events could get into the energy spectrum. Correspondingly, some fraction of the fastest rise-time neutrons were rejected. The combination of these two effects is expected to be the cause of the dip in the energy spectrum at channel 120. For this reason, the neutron calibration directly uses the counts in the neutron peak of the rise-time spectrum and not the gated energy pulses.

With 3 -hour counting times there were modest background counting rates. The background rates are shown in Table 2, and have been subtracted from the raw data yielding small (less than 1\%) effects for the NE213 DT data and modest (ten percent) corrections for the NE213 DD data.

\section{2. ${ }^{3}$ He Energy Spectra}

The ${ }^{3}$ He ionization chamber spectra obtained under different circumstances including a series of TFTR plasmasi2] are shown in Figure 10. The spectra are composed of a pulser (channel 370) which monitors the integrity of the electronic pulses, a $2.45-\mathrm{MeV}$ neutron peak (channel 195) which is Doppler broadened by the $2.5 \mathrm{keV}$ 


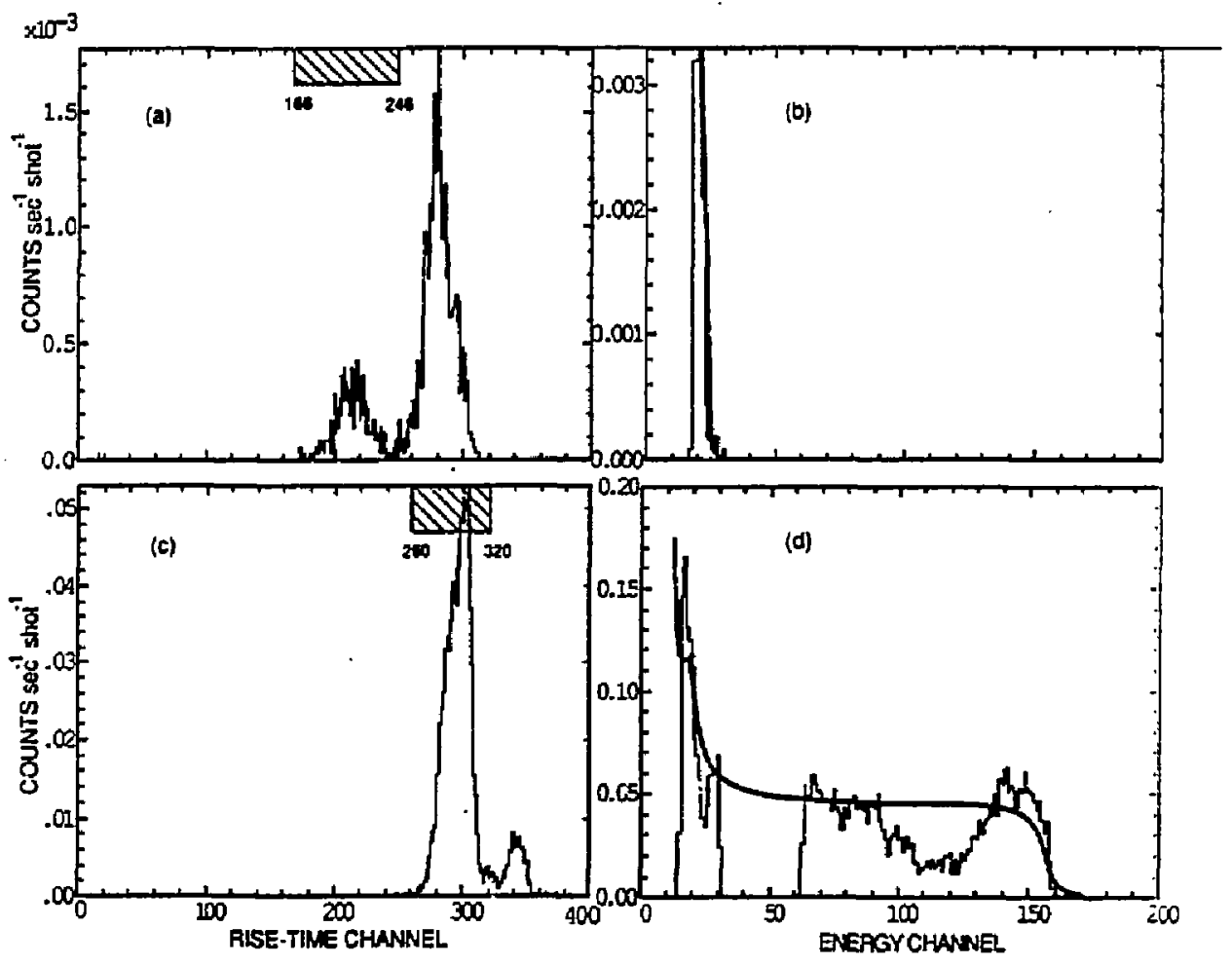

Figure 9: Histograms of the NE213 detector response to the DD (a and b) and DI ( $c$ and d) generators. (a) and (c) are the rise-time spectra, and (b) and (d) are ibe energy spectra. Each spectra are integrated over several shots. The solid line in (d) is the expected detector response, normalized to the virgin neutron recoil edge. The hatched regions show the neutron windows used. 
ion temperature of the plasma deuterons, some low energy neutron counts down to about 1.7 MeV, a recoil proton edge due to methane gas in the jonization chamber, a ${ }^{3} \mathrm{He}$ recoil proton edge, a thermal ${ }^{3} \mathrm{He}(n, p) t$ peak at channel 52 , and a low energy exponential spectrum probably due to $\gamma$ induced counts.

The calculated detector response function[18! (Figure 11) for pure 2.5-MeV neutrons is similar in shape and relative magnitude to the measured spectrum above 2 $\mathrm{MeV}$ (channel 160) indicating that most of the counts between the 2.5-MeV Doppler brozdened peak and proton recoil edge are wall counts. Wall counts are events where one of the ${ }^{3} \mathrm{He}(n, p) t$ products, usually the proton, has lost energy to the walls. A prominent aspect of the response function is the ${ }^{3} \mathrm{He}$ recoil edge iFigure 12(a)]. Its magnitude might be useful in establishing the absolute calibration of the detector; however. pulse pile-up of the thermal neutron counts with themselves (channel 95 in the measured spectrum) is a noise source for determining the magnitude of the ${ }^{3} \mathrm{He}$ recoil edge. The recoil edge in the $3 \mathrm{hr}$ spectrum is also lower than for the plasma case which we interpret to be a combination of the plasma having more lower energy scattered neutrons as well as more pulse-pile up of the thermal neutron peak with itself. The detector response function has no calculated response to epithermal neutrons; however, the plasma spectrum does indicate the presence of epithermal neutrons and low energy ( $<1.5 \mathrm{MeV}$ ) neutrons. These are scattered neutrons that might originate either at the vacuum vessel or inside the collimator.

Since we have taken a three hour run with the DD neutron generator in the Bay $F$ plasma location, we also took a three hour exposure without any source operating. This background spectrum (shown in Fig. 12) did not indicate any epithermal neatron events but did indicate a significant background $\left(10^{-1} \mathrm{cps}\right)$ in the vicinity of $2.5-\mathrm{MeV}$ neutron energy. Presumably, these events are cosmic ray events or possibly some internal arcing in the detector or in the cable or connectors.

The entire spectrum for the three hour run is plotted in Fig. 10(a) with details shown in Fig.12. An epithermal neutron peak is clearly evident above background as is a ${ }^{3}$ He recoil edge (Fig. 12(a)). The 2.5-MeV neutron peak (Fig. 12(b)), however, is only marginally evident above the background.

The spectrum obtained when the DT neutron generator was used $F i g .10(c)$ j shows no observable peak since it would be off-scale. Furthermore, above about $3 \mathrm{MeV}$ neutron energy, the detector response becomes dominated by wall events. That is, the ${ }^{3} \mathrm{He}$ recoil and ${ }^{3} \mathrm{He}(\pi, p) t$ reaction products have so much energy that they tend to strike the wall. Therefore, we take the detector response in channels 60 to 200 to be dominated by ${ }^{3} \mathrm{He}$ recoils which, however, do not go to full energy because they strike the wall. 

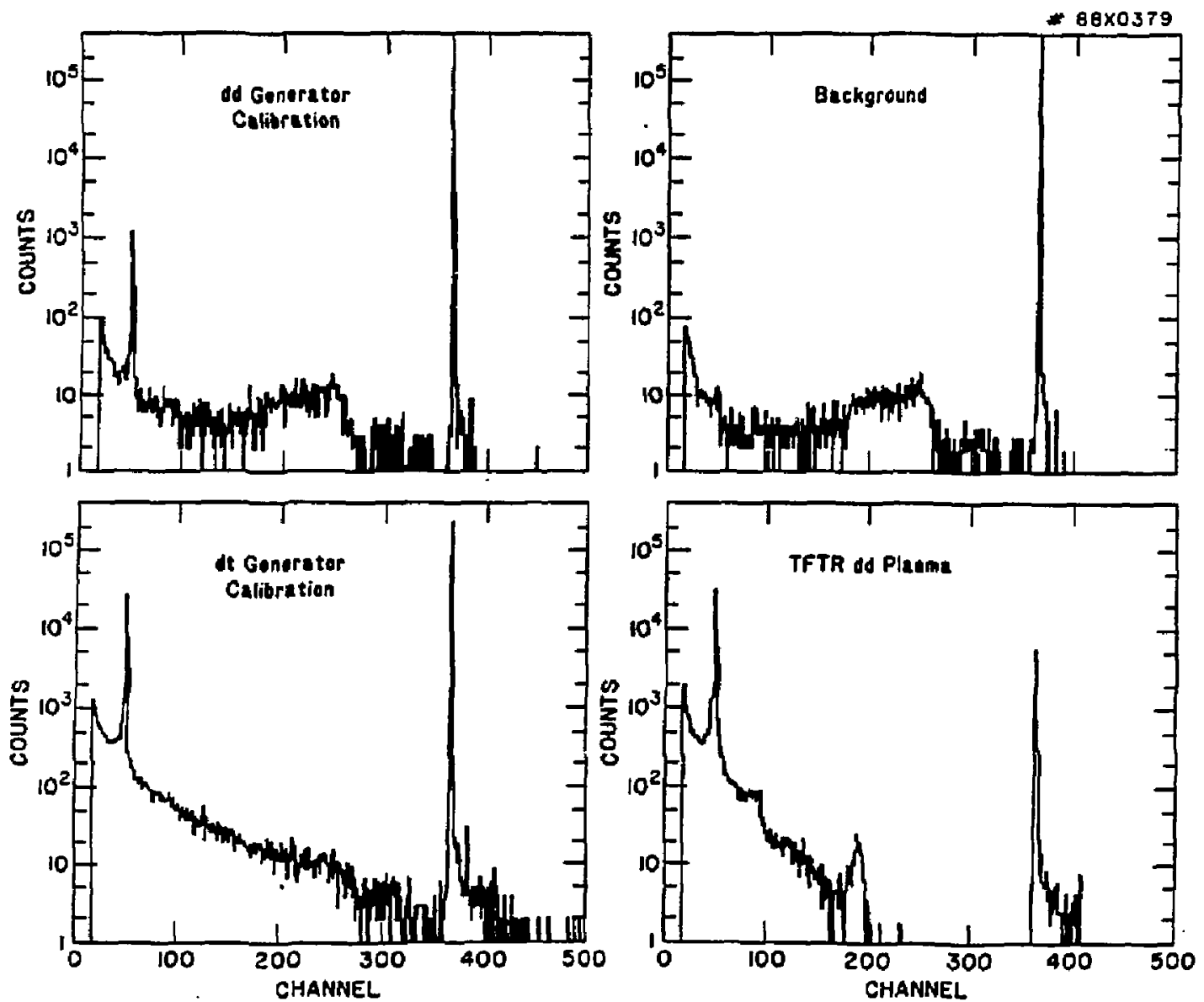

Figure 10: The ${ }^{3}$ He jonization chamber energr spectra of the 3 hour DD generator run, a 3 hour background exposure. a 16 minute DT generator run. and TFTR deuterium ohmic plasmasi?. 

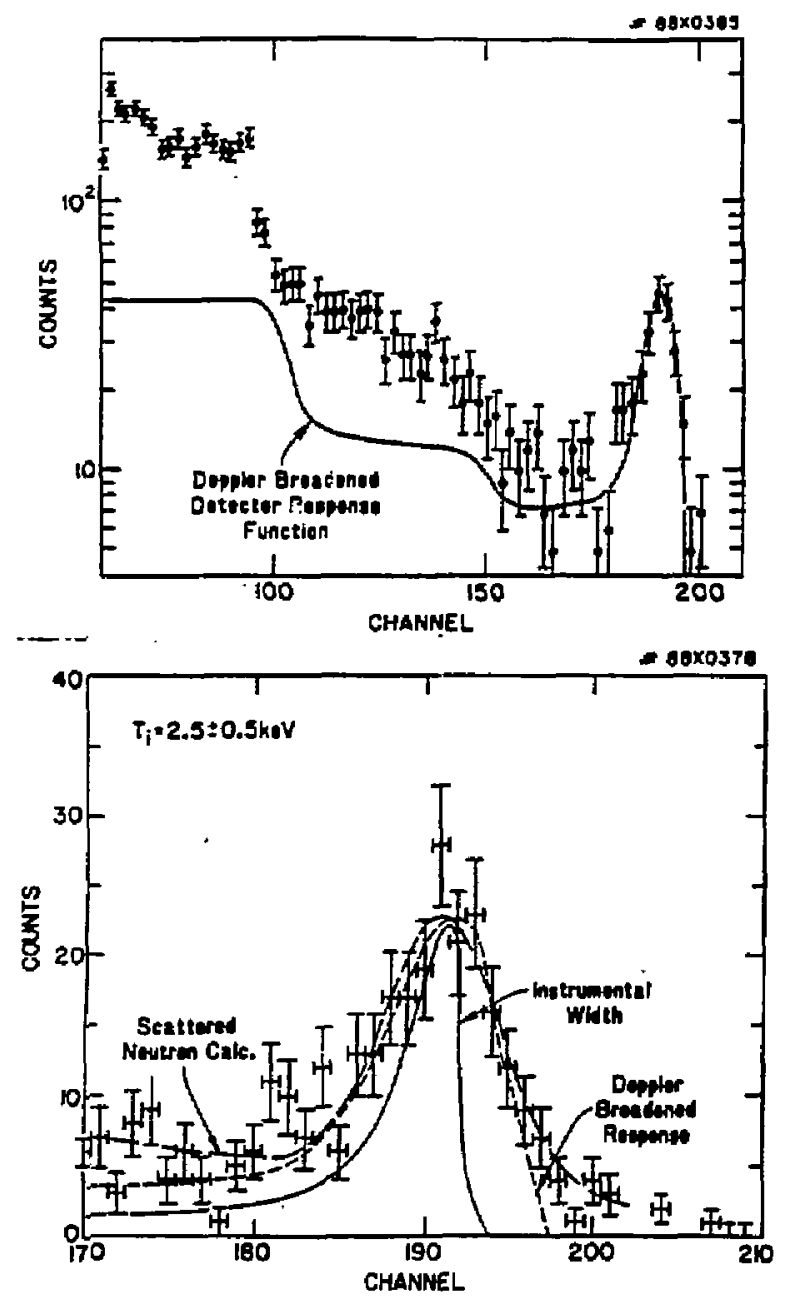

Figure 11: The ${ }^{3} \mathrm{He}$ ionization chamber spectrum for the 20 TFTR deuterium ohmic plasmas 2 and the detector response function 18 to a pure $2.5-M e V$ neutron spectrum doppler broadened and normalized $u$ the plasma spectrum at 2.5 Mel...(Top) Data (solid points) and response function over the entire energy range. (Bottorit) Data near $2.5 \mathrm{Mel}$ (error bars), the instrumental response function $1 \mathrm{~d}$ (solid line), the Doppler broadened response function (short dashed line). and the fit to the data assuming scattered neutrons accounted for the lower energy neutrons (long dashed line) 1. 

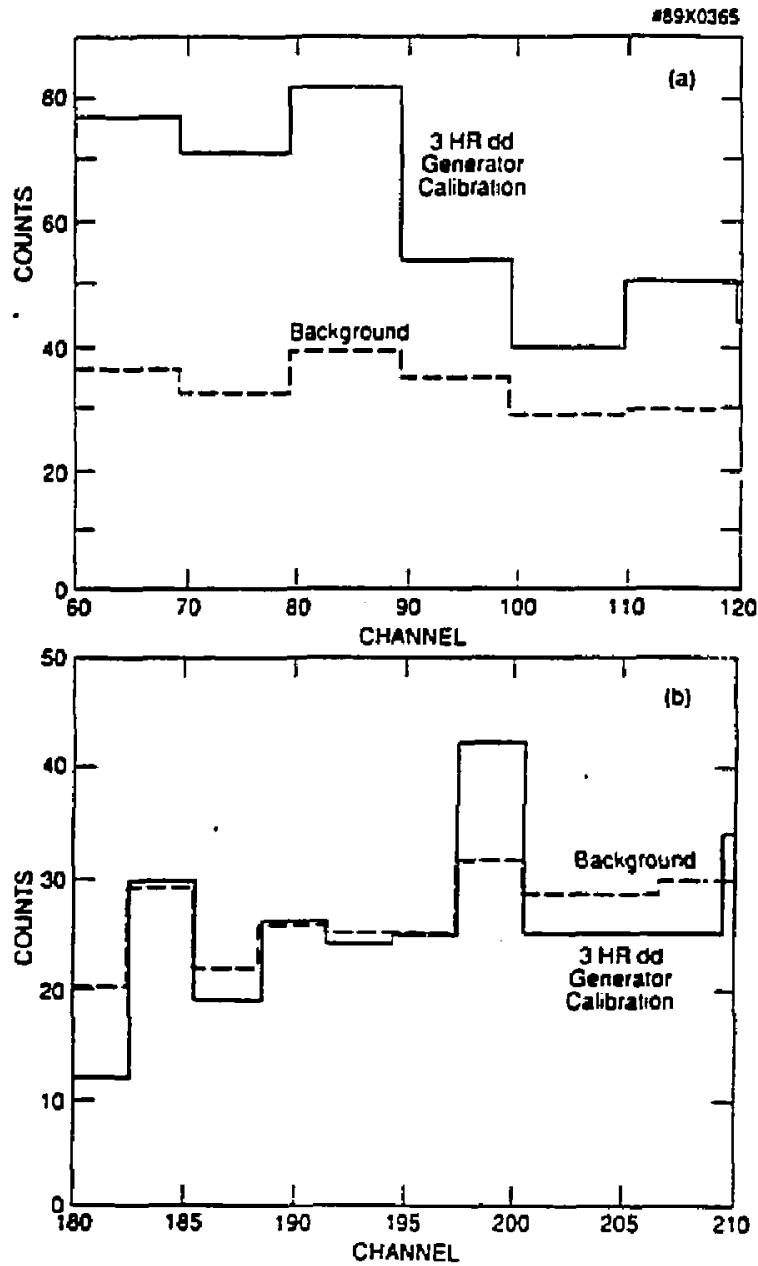

Figure 12: The 3-hr generator-Iun and background spectra in the vicinity of: (a) the ${ }^{3} \mathrm{He}$ recoil edge. and (b) the 2.5-Mel' peak. 


\section{Collimator Aperture Map and Detector Efficiencies}

\section{1. .VE213 Detector}

\section{f.1.1. Aperture Maps}

The point efficiencies $\varepsilon$ versus toroida] angle $\phi$ for the DT [Figure $13(\mathrm{a})$ ] and DD Figure 13(b) / generators for the NE213 system were peaked near the center of Bay $F$. They show similas features, approximntely at the locations of the toroidai field coils and, with the Bay $F$ view, the thick flanges on the Bay $F$ port. The data taken at higher z-positions and with the generator rotated outwards by $45^{\circ}$ is shown in Fig. ure 14 . Such data is about equivalent to data taken at toroidal angles $z(360 / 2 \pi) / R_{0}$ which are $3^{\circ}$ or $6^{\circ}$ from the peak. Data from both generatcrs was taken on the far side of the machine from $198^{\circ}-270^{\circ}$ in toroidal angle, but at significantly reduced generator emission for the DT case. In both cases, the detector point efficiencies, when corrected for background, were consistent with being zero. For example, with the DT generator 7 counts were seen with an expected background of 8.6 counts but for a total yield of only $1.9 \times 10^{\text {it }}$ neutrons.

Typical profiles of neutron emission calculated for TFTR ohmically-heated or bearn-heated plasmas are sufficiently narrow that most of the neutron emission would fall inside the collimator aperture (Figure 15). Moreover, the vertical alignment (within $10 \mathrm{~cm}$ ) is probably sufficient to ensure that both the ${ }^{3} \mathrm{He}$ ionization chamber and the NE213 detector observe over $85 \%$ of the neutron emitting plasma (Fig. 15). Since the calibration demonstrated significant local neutron absorption and scattering, future calibrations should attempt a turo-dimensional map of the area.

\subsubsection{Neutron efficiencies}

There are $10-20 \%$ uncertainties, largely from generator uncertainties, in estimating the point efficiencies of the peak (in units of counts per neutron)

$$
\begin{aligned}
& \epsilon_{D T} \text { (peak) }=5.4 \times 10^{-10} \\
& \epsilon_{D D}(\text { peak })=1.2 \times 10^{-10}
\end{aligned}
$$

and at $-2^{\circ}$ (where the 3 hour exposure was taken)

$$
\begin{aligned}
& C_{D T}\left(-2^{\circ}\right)=4.3 \times 10^{-10} \\
& \epsilon_{D D}\left(-2^{\circ}\right)=6.8 \times 10^{-11} .
\end{aligned}
$$

To get a tnroidal angle average efficiency for comparing to a line-source of neutrons, one can linearly interpolate through the point efficiency data (or draw a smooth 

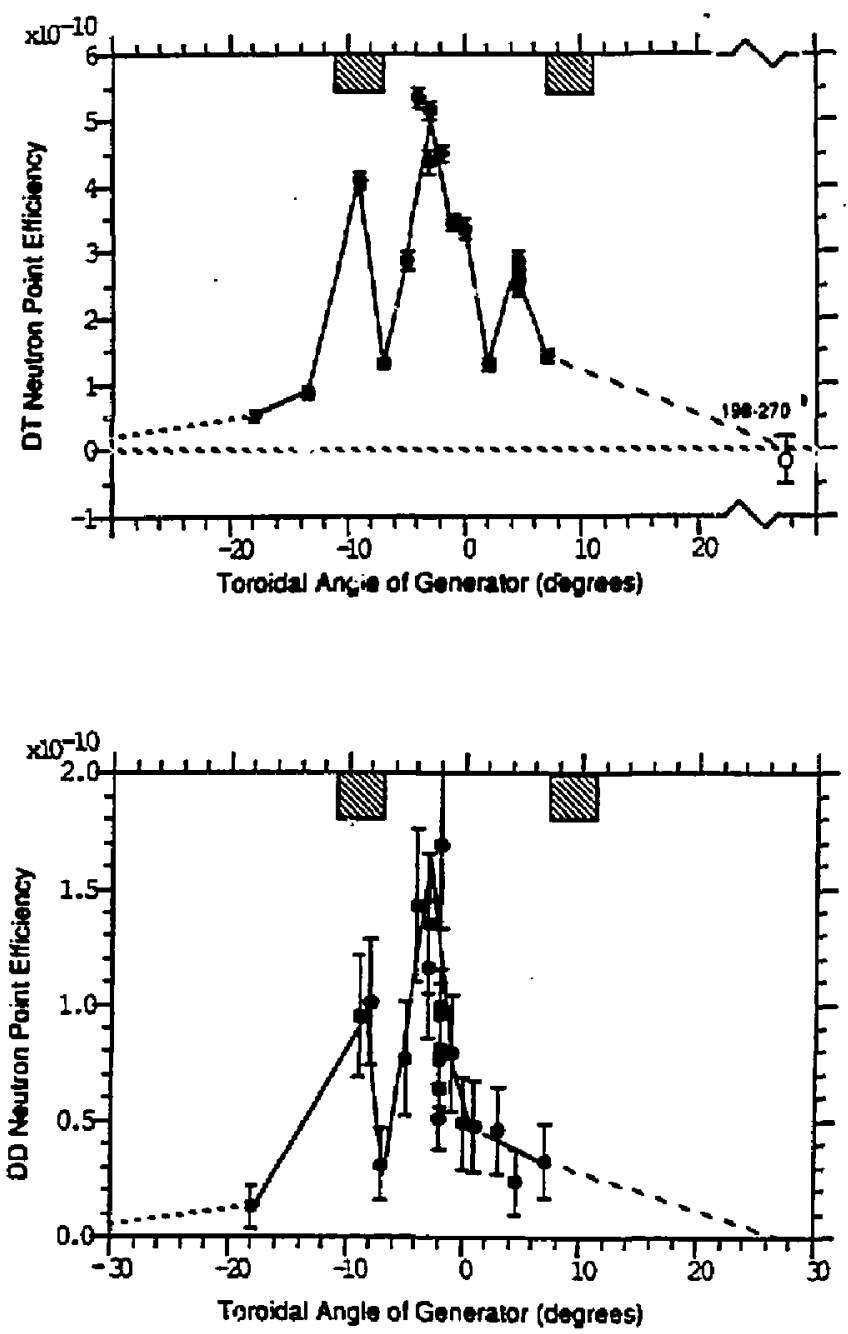

Figure 13: Point efficiency iersus toroidal angle for the NE213 detector. (a) Response to the DT generator. (b) hesponse to the DD generator. The hatched regions are centered at $=9^{\circ}$ to illustrate about where the TF coils define the bas. 


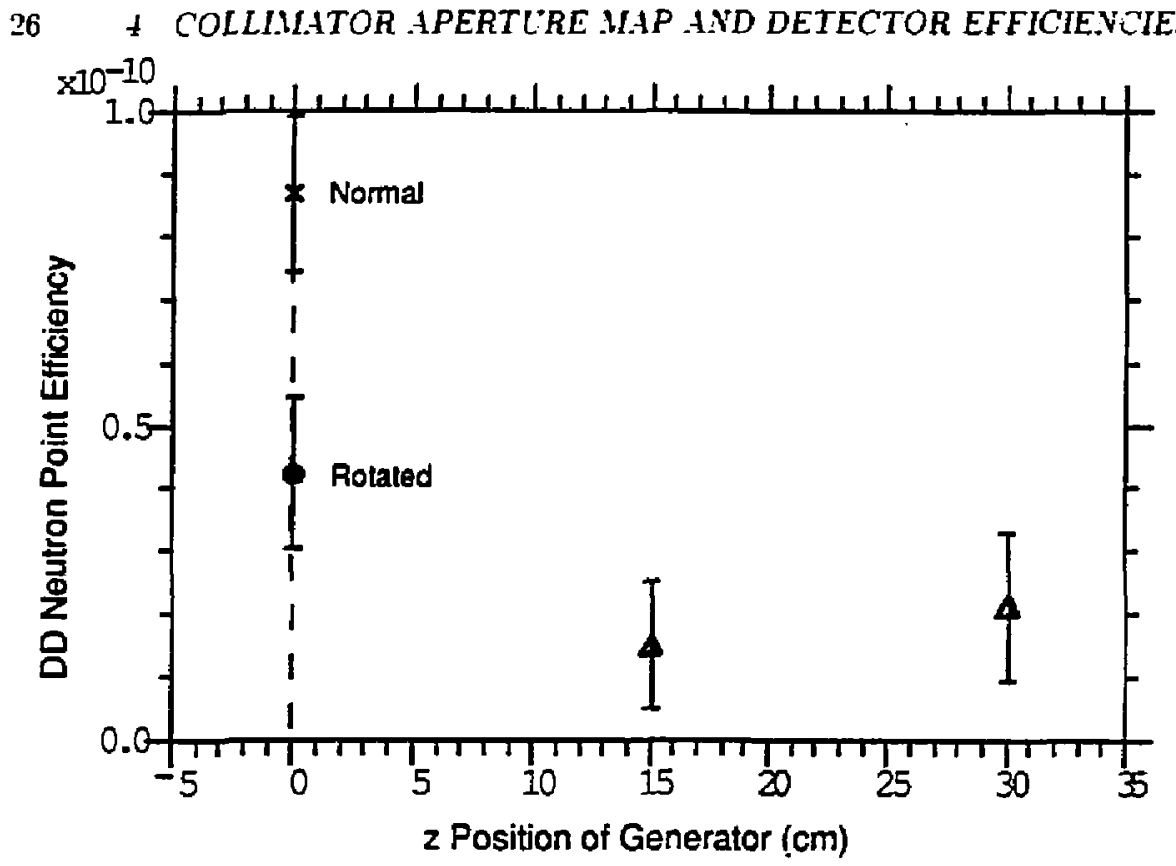

Figure 14: Point efficiency versus z-position of the DD generator for the NE213 detector. Al] data was taken at $-2^{\circ}$ angle. The open circle represents the shot with the generator rotated.

curvel. and then integrate the area. That is, the detector efficiency to a turoidal linesource is

$$
\eta=\frac{I}{\int d \phi}=\frac{\int \epsilon d \phi}{\int d \phi} .
$$

One can divide the integral $I$ by the point efficiency $\epsilon$ somewhere to estimate the "average" width in angle of the data.

One question is the contribution to the integrated efficiencr from data at angles not measured. In 1985. a ${ }^{252}$ C $f$ source was moved inside TFTR and observed by a XE213 detector in the neutron collimator in approximately the same location. In retrospect insufficient toroidal detail was taken in 1985 to clearly resolye the peak. and there were questions about the detector background. The 1985 point efficiency data uncorrected for any background decreased to a toroidally constant level by about 2 bays $\left(36^{\circ}\right)$ from Bay $F$ on either side. In treating the 198 it data. We assumed that the point efficiency of our detector is zero on the far side of the machine, and that the 
14 degree aperture assumed
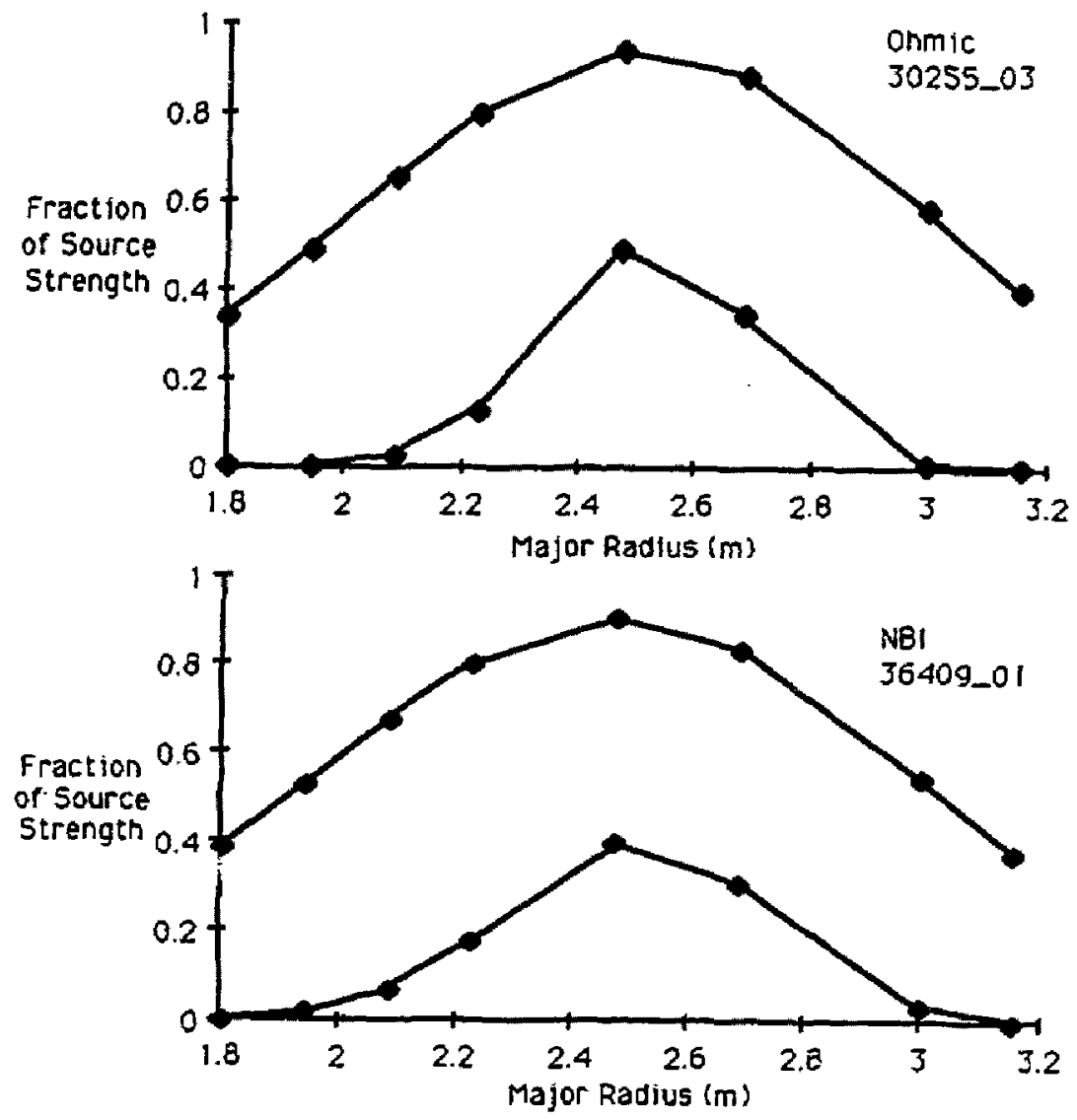

Figure 15: Calculations of collimator view of neutron profiles. The closed symbols represent the calculated (by S.A.AP) line-integrated neutron emission profile. The open symbols represent the fraction of the total emission seen by a colltmated detector if the center of its view was at the labeled major radius. The calculation assumes the collimator aperture has a Gaussian profile with an equivalent width of $14^{\circ}$ of toroidal angle, and convolutes that aperture with the line-integrated emission. The calculation thus assumes that vieving the plasma from below is equivalent to viewing the plasma from the outside horizontal midplane. (a) The ohmic TFTR plasma (shot 3025.5 try 03) from which the neutron spectrum was measured 2. (b) A neutsal beam injerted plasma of 1.8 Af and 2.7 MIW (shot 36409 try 01 ). 
point efficiency drops linearly to zero from the measured end points within $1=0$ : bay.

The detector efficiency to a toroidal line-source, $\eta(E \mathrm{~g} .2)$, again in units of counts per neutron, is the sum of the integrals over peak region where the measurements were taken and over the wings where littie data was taken, $\left(I_{\text {peak }}+J_{\text {wingos }}\right) / 360^{\circ}$. This is

$$
\begin{aligned}
\eta_{D T} & =2.1 \pm 0.4 \times 10^{-11} \\
\eta_{D D} & =4.4 \pm 1.3 \times 10^{-12}
\end{aligned}
$$

where the $12 \%$ error on the generator direct emission is included. We estimate about $10 \%$ overall accuracy in integrating the available data for the DT neutrons, and a $20 \%$ error in determining the shape and a $10 \%$ error in determining the overall magnitude for the DD data. The ratio of these efficiencies is $4.8 \pm 1.0$ where the uncertainty of the ratio is reduced by temoving similar systematic uncertainties. The average width of the aperture $I / c$ (peak) is about $14^{\circ}$.

The DD and DT angular struetures are consistent with being the same within the $12 \%$ uncertainties of the generittor emission. There were 6 pairs of toroidal angles with data from both generators with more than 9 counts above background for the $D D$ generator. The average of the ratio of point efficiencies $E_{D T} / \epsilon_{D D}$ for these 6 pairs of toroidal angles is $4.9 \pm 0.6$. The DD data at $-2^{\circ}$ is low (the efficiency ratio is 6.0 ) but still statistically consistent with the other ratios.

The major contribution to the uncertainties in the NE213 calibration come from the accuracy of the toroidal integrals through the sparse data set. Significant variations in the point efficiency with angle wete seen, raising questions about further unmeasured variations in spaces between the data points that were sampled. However we feel that our quoted uncertainties include the possibility of such variations as well as the statistical errors, that they are relatively conservative and, when doubled, that they tepresent a high (95\%) level of confidence of the accuracy of the measurement.

The observed fraction of low-energ: channel counts for DT operation was 4505 out of 12220 seen in the high energy channel, or $37 \%$. This was near the $31=4 \%$ estimated from the ratio of counting efficiencies, as expected from the NE213 detector response function[13].

The expected efficiency for the XE213 system in the absence of any attenuation or scattering was the geometric factor (Eq. 1) times the counting efficiency. For DT תeutrons this would be

$$
J_{\text {expered }}=5.7 \times 10^{-2} \times 3.7 \times 10^{-8}=2.1 \times 10^{-8} \text { counts per neutron }
$$

which is 100 times greater than observed. The expected point efficiency in the center was $\left(A / 4 \pi L^{2}\right) 5.7 \times 10^{-2}=4.3 \times 10^{-8}$ instead of the $5.4 \times 10^{-30}$ observed, again the 
Table 3: Materials between the plasma and the spectrometers in the collimator.

\begin{tabular}{l|rrrr} 
Materjal & Steel & Inconel & Carbon & Lead \\
\hline SpecificDensity $\rho$ & 8.02 & 8.25 & 2.0 & 11.3 \\
Atomic Weight $A$ & 58 & 58 & 12 & 207 \\
Thickness (inches) $t$ & 0.38 & 3.0 & 1.5 & 2.0 \\
$\sigma_{r}(15 \mathrm{MeV}$ ) (barns) & 1.55 & 1.55 & 0.92 & 3.39 \\
$\sigma_{r}(3 \mathrm{MeV}$ ) (barns) & 1.95 & 1.95 & 1.58 & 3.72 \\
ptor $/ \sigma_{r} / m_{p}(15 \mathrm{MeV})$ & 0.13 & 0.98 & 0.35 & 0.57 \\
pt $\sigma_{r} / A m_{p}(3 \mathrm{MeV})$ & 0.16 & 1.24 & 0.60 & 0.62
\end{tabular}

same two orders of magnitude low. The reduced efficiency can be partially explained by effects of small-angle scattering between the plasma and the detector. The "removal cross-section" represents the probability per atom of a scattering event that changes the energy of the neutron, followed by its subsequent removal by moderation in hydrogenous material (such as the paraffin of the collimator)[19]. Table 3 lists the approximate materials between the detectors and the neutron source, their remotal cross-sections at 3 and $15 \mathrm{MeV}$, and their estimated effect on the "attenuation" of the directed neutrons. The average thickness of the Inconel of the port is uncertain and could be from 1 to 5 inches in places. The carbon of the protective plates is an important effect, for despite the lower removal cross-section it has a high number density $\rho / A m_{p}$. Summing up the removal coefficients $\rho t \sigma_{r} / A m_{p}$ and exponentiating the sum gives the estimated effect of the attenuation to the ${ }^{3}$ He detector. Adding the effect of the additional 2 inches of lead in front of the NE213 delector yields a total factor of $1 / 13$ for $D T$ neutrons and $1 / 26$ for $D D$ neutrons to that detector. These calculated effects are about one-thitd to one-quarter of that seen in the calibration. The 2.5-MeV neutron response is expected to be a factor of 4 lower than the $14-$.11el response, half because of differential attenuation and half because of the factor of 1.9 difference in counting efficiency, with no effect from differences in the angular acceptance of the collimator. In view of the distance of the collinator to the plasma and the variability of materials in that distance, we take these attenuation factors as reasonable agreement with the observed $1 / 100$ attenuation.

Comparing the energy spectra (even with the $\gamma$ problem described above) for data taken looking at $99^{\circ}$ in the forward direction with respect to the generator as opposed to $73^{\circ}-83^{\circ}$ in the backward direction shows about a 2 channel shift of the recoil edge. This shift is consistent with the $0.2 \mathrm{MeV}$ change in generator energy between those angles[20]. Comparing data taken in the peak of the aperture map with 
data taken in the xings shows no difference in the shape of the spectrum attributable to scaltering.

The effects of scattering on the response have been studied in another way. A sequence of 32 Ohmic discharges run with helium had a very low neutron rate. From 1-3 seconds a total of 3 neutron counts and $19 \gamma$ counts were seen by the NE213 system. From 3-5 seconds the neutral bears were injecting into thejr calorimeters, causing a background of scattered neutrons. During this time $3375 \gamma$ counts were seen, and 28 "neutron" events, or a $0.83 \%$ background from the $\gamma$ counts. In typical deuterium piasme discharges the neutron counts are about $39 \%$ of the number of $\gamma$ counts. Hence, if we make the assumption that the calorimeter neutrons make the same fraction of 75 at the NE213 detector as the plasma neutrons do, then the normal component of scattered neutrons to the NE213 system is less than $0.0083 / 0.39=2 \%$.

The single experiment of rotating the DD generator towards the detector resulted in a factor of two drop in the point efficiency (the detected counts dropped while the directed yield increased from the rotation) (see Fig. 14). Only 13 counts were observed, and about 30 were expected. However, it was difficult to insure the toroidal angle of the generator bead was exactly the same when the entire generator was rotated. Small positioning errors at the -20 location of the experiment could have had a large impact on the results because of the steep gradient of the point efficiency at that angle (presurably because of shielding by the edge of the flange).

\subsubsection{Comparison of yields to other calibrated systems}

The neutton yields from the NE213 detector compared to the fission chamber system 9] are within statistical accuracy of 1.0 for DD neutron source strengths from $5 \times 10^{12} \mathrm{sec}^{-1}$ in Ohmic discharges to $6 \times 10^{14} \mathrm{sec}^{-1}$ in NBI discharges (Figure 16). There is no discrepancy on the overall DD neutron calibration within the $30 \%$ uncertainty of the NE213 calibration. This is despite the ${ }^{225} \mathrm{l}:$ fission chamber primarily responding to seattered neutrons of all energies, while the NE213 responds to collimated, unscattered, "directed" neutrons of nearly full energy. The relative precision of comparing discharges is about $10 \%$ from counting statistics in the NE213 and the accuracy of identifying all the reutron counts without a gamma contribution. Yo dependence on major radius between $2.45 \mathrm{~m}$ and $2.60 \mathrm{~m}$ is seen within that relative precision between discharges, and no difference on whether the neutrons are from ohmic or neutral beam injection. This last point differs from previous'results with the ${ }^{3} \mathrm{He}$ detector 3 ? where a factor of 1.5 was seen between ohmic and certain special neutral beam discharges. A slow decrease in the efficiency of the ${ }^{238} \mathrm{U}$ detector can be seen in this comparison, confirmed by comparison to other detector systems. Table 4 shows the shot lists used for the comparison. 


$\begin{array}{ll}\text { Lebel } & \text { Expression } \\ \text { NE213DDEFF } & 4.4 E-12 \\ \text { NE2DDEFF } & \text { 3.AE-9 } \\ \text { NEEDOEFF } & \text { 2.ADE-11 }\end{array}$

Descriptions:

Crose Colibration Dutabose of NE233 and ME2 and MES

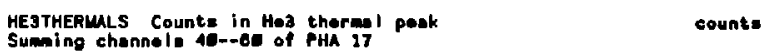

NE2 counte in NE2 counts

Pile-up corrected for 31786 with $1.06-6$ soc dead-time.

NE213 Integrated counte in NE213

counte

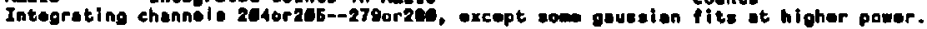

Database: CROSS_NES Relatione: CROSS_NES O-FED-to 10:14:48 Page 1

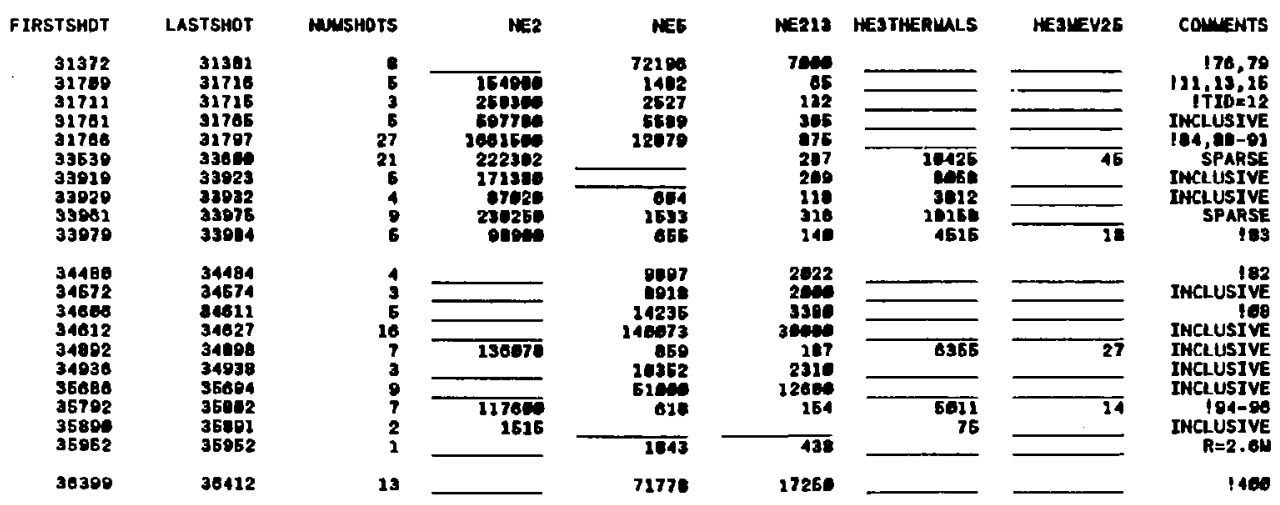




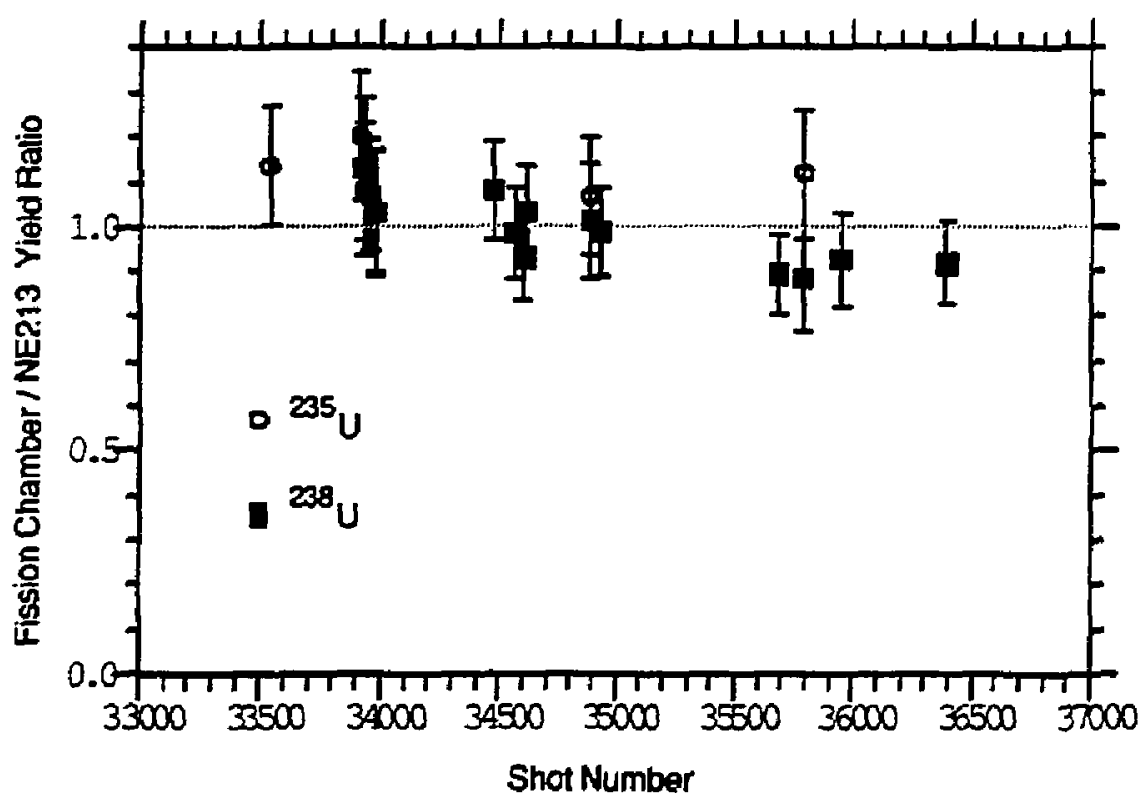

Figure 16: NE213 yield divided by NE system yield versus shot number. The open symbols are for the ${ }^{235} \mathrm{U}$ detector (NE2) and the closed symbols for the ${ }^{230} \mathrm{U}$ detector (NE5). For the 200 /NE213 cornparison both ohmic and neutral beam heated discharges are included, as well as discharges at $2.45 \mathrm{~m}$ and $2.6 \mathrm{~m}$ major radius.

The $\gamma$ signal in the high energy circuit during the DD generator calibration was statistically consistent with being entirely due to background, with 1376 events seen compared to a background expected of 1340 events. However, this statistically insignificant difference of 36 out of 1340 counts (one sigma increase), if continued uniformly from neutrons erritted around the whole torus, would lead to a detection efficiency of $\gamma$ 's in the DT channel of $8 \times 10^{-12}$, which is actually greater than the neutron efficiency of the low energy channel, $\eta_{D D}$. That is, we could not determine directly the detection efficiency to $\gamma$ 's in the DT channel. In actual präctice we see almost the same number of $\gamma$ events in the DT channel as neutron events in the DD channel from TFTR plasmas. With a better than 100-1 $\gamma$-rejection in the DT circuit and the ratio of DT/DD efficiency for neutrons of 4.9 , this provides a conservative lower bound for the burnup to be detected of $1 /(100 \times 4.9)=0.2 \%$. Integrated burnup of about $0.3 \%$ can be clearly detected by a peak in the $14 . \mathrm{MeV}$ neutron 
rise-time spectrum. After the neutral beam injection the amount of $\gamma$ 's drops more rapidly than the $14-\mathrm{MeV}$ neutron emission, and the detector easily becomes sensitive to much lower emission levels.

The DT neutron source strength from the NE213 system is consistent with the rate deduced from the ${ }^{235} U$ fission chamber using its DT calibration[9,8] (Figure 17). However, since the exact ratio of DT/DD relative emission at these late times is unknown, it is possible that the DD rate is still up to $50 \%$ of the DT ralei8]. $50 \%$ DD rate (1 out of 3 geutrons) with a factor of 5 lower detection response than for the DT's in the NE213 system means only one-tenth as many DD events as DT events at this time. And since in the low energy circuit there is 1 event for every 3 in the high energy channel just from DT neutrons, one is faced with subtracting 10 counts for every 13 in the low energy channel to delermine the actual DD rate. Only with large numbers of counts from many reproducible discharges to reduce the statistical error can this be doae accurately. While from the NE213 detector it appears that the DD rate is much less than $50 \%$ of the DT rate, this result is within the $40 \%$ uncertainty of the relative DT/DD calibration of the ${ }^{225} \mathrm{U}$ detectors which found the high level of DD neutrons[8]. This would then mean that there could be a $50 \%$ difference between the fission chamber DT source strength and the NE213 DT calibration.

\section{2. ${ }^{3} \mathrm{He}$ Detector}

\subsubsection{Aperture map and scattering}

For the ${ }^{3}$ He detector the aperture was mapped using the DD and DT generators with the thermal ${ }^{3} \mathrm{He}(n, p) t$ counts, and with the ${ }^{3} \mathrm{He}$ recoil counts. The ${ }^{3} \mathrm{He}$ recojl counts for the DT generator scan (Figure 18) yield the best statistics. The shape of the aperture map is very similar for the ${ }^{3} \mathrm{He}$ detector and the NE213 detector, but with differences attributed to scattering. Neutron penetration through the collimating cones might further broaden the optically expected response. According to such a model, the aperture view should be flat between the toroidal field coils and cut off by the toroidal field coil (Fig. 18). The observed collimator aperture is narrower than the distance between the toroidal field coils and shows local absorption efiects in the vicinity of the flange edges (6 inches of steel) (see Fig. 3). The ${ }^{3} \mathrm{He}$ recoil mapping and the NE213 recoil mapping (Fig. 18) follow each other except in the wings where the ${ }^{3} \mathrm{He}$ recoil signals are relatively higher. Since the ${ }^{3} \mathrm{He}$ recoils are at a lower energy relative to the fecoil edge than are the NE213 signals, there may be more scattered neutrons of degraded energies in the ${ }^{3} \mathrm{He}$ recoil counts. Consequently, the wings would have a larger component of scattered neutrons causing the relatively larger ${ }^{3} \mathrm{He}$ detector response. 


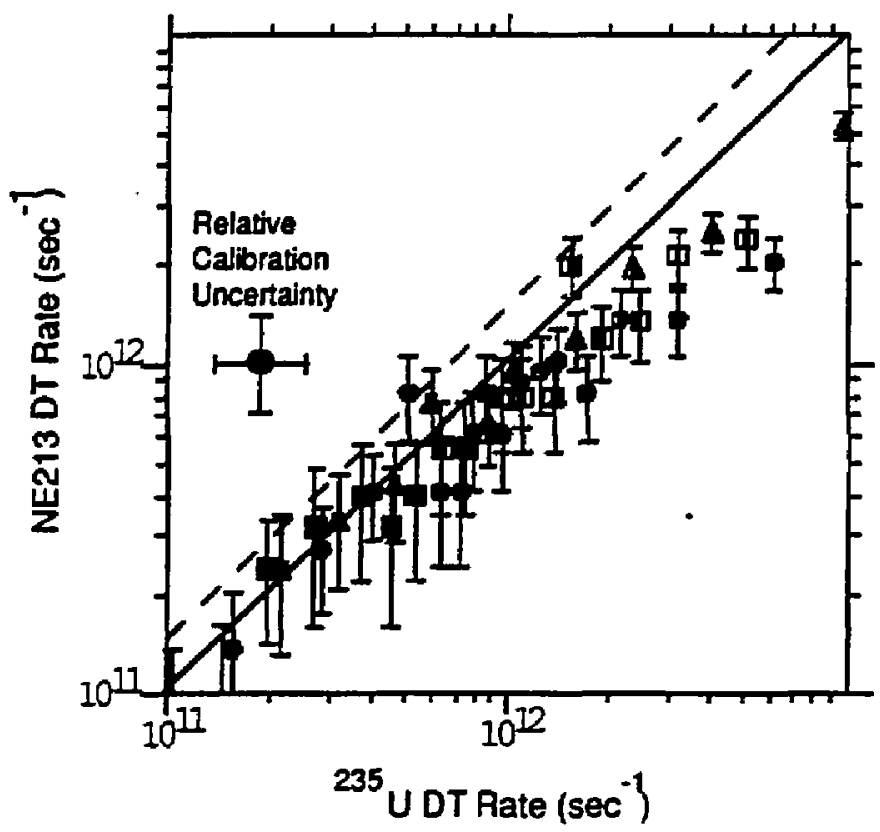

Figure 1i: The XE213 DT neutron source strength is plotied versus the rate from the NE2 detector (assuming all the neutrons are DT). The data are from the arcumulations of three sequences of discharges during the post-NBI phase. If the DD rate were still $50 \%$ of the DT rate the data would shift left by the distance from the solid to the dashed line.

Some features of the aperture scan data give an indication of the relative proportions of scattered neutrons deterted inside the collimator:

1. The thermal (multiple scattered) neutron counts 'Figure 19(a)' have a similar aperture map to the more energetic ${ }^{3} \mathrm{He}$ recoil neutron counts, except in the wings where there is more response to thermal neutrons. This indicates that a significant proportion of the thermal neutron counts in the detectpr originate from already scattered neutrons entering the aperture.

2. The dip at the flange edge $\left(-6^{\circ}\right.$ toroidal angle point) indicates that many of the thermal neutron counts also originate as direct neutrons and thus have been thermalized inside the collimator. 


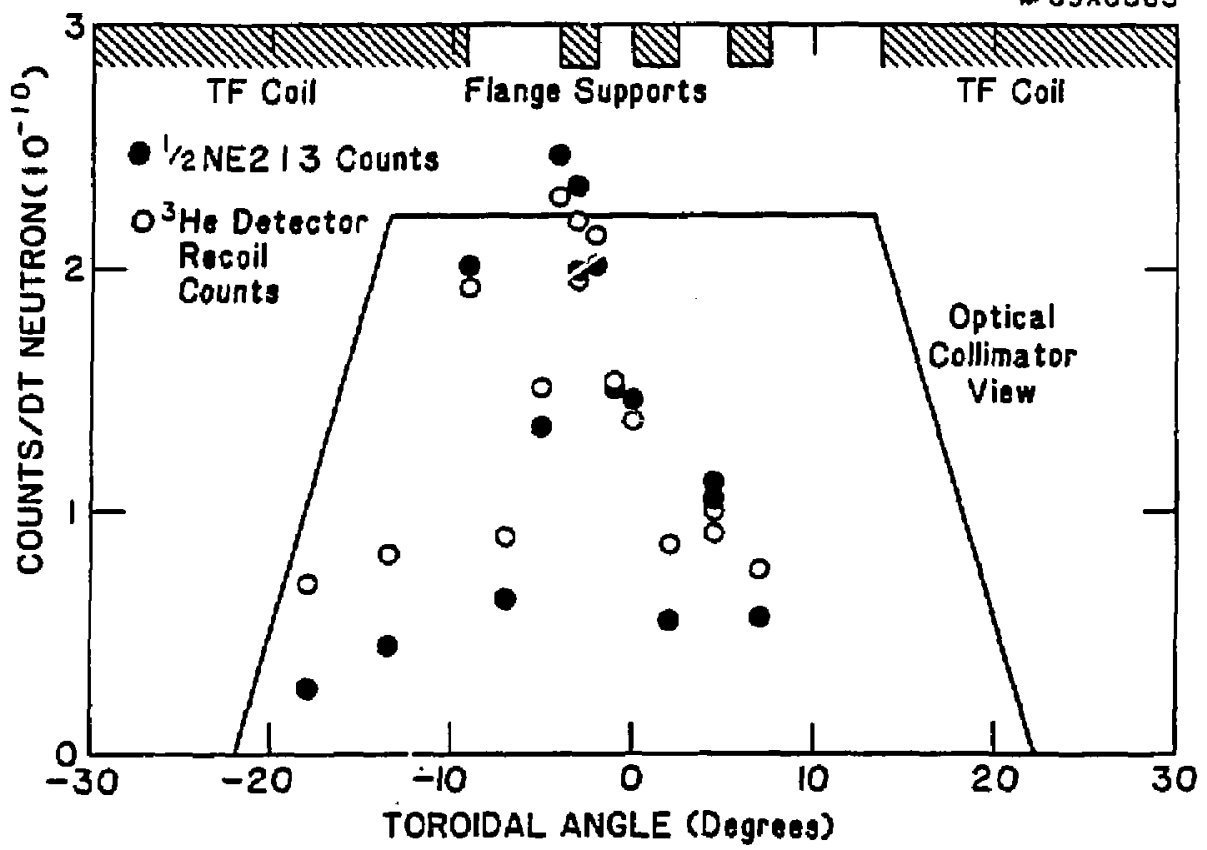

Figure 18: The ${ }^{3}$ He recoil counts for the Bay $\mathrm{F}$ scan of the DT generator, compared to the $\mathrm{XE} 213$ response. The optical view of the collimator is approximately $=10^{\circ}-12^{\circ}$.

3. The detected thermal counts per emitted neutron were lower with the DD generator than with the DT generator 'Fig. 19(a):. This would indicate that the 14-Mel neutrons caused a larger, scattered neutron flux inside the collimator. Probably the higher neutron energy of the DT neutrons penetrated the collimator aperture more easily. However. such penetration causes enough energy degradation that the high energy threshold of the XE213 detector still results in similar angular maps for DD and DT.

The aperture map Figure $19(b)$, of the ${ }^{3}$ He recoil edge for the DD neutron generator again indicates a similar aperture to the DT generator. However. the count rates are so low that the statistical uncertainties make this map less useful than Fig.19(a). Nie do :ot attach any significance to the high point at $-18^{\circ}$ because of its large statistical uncertainty. 

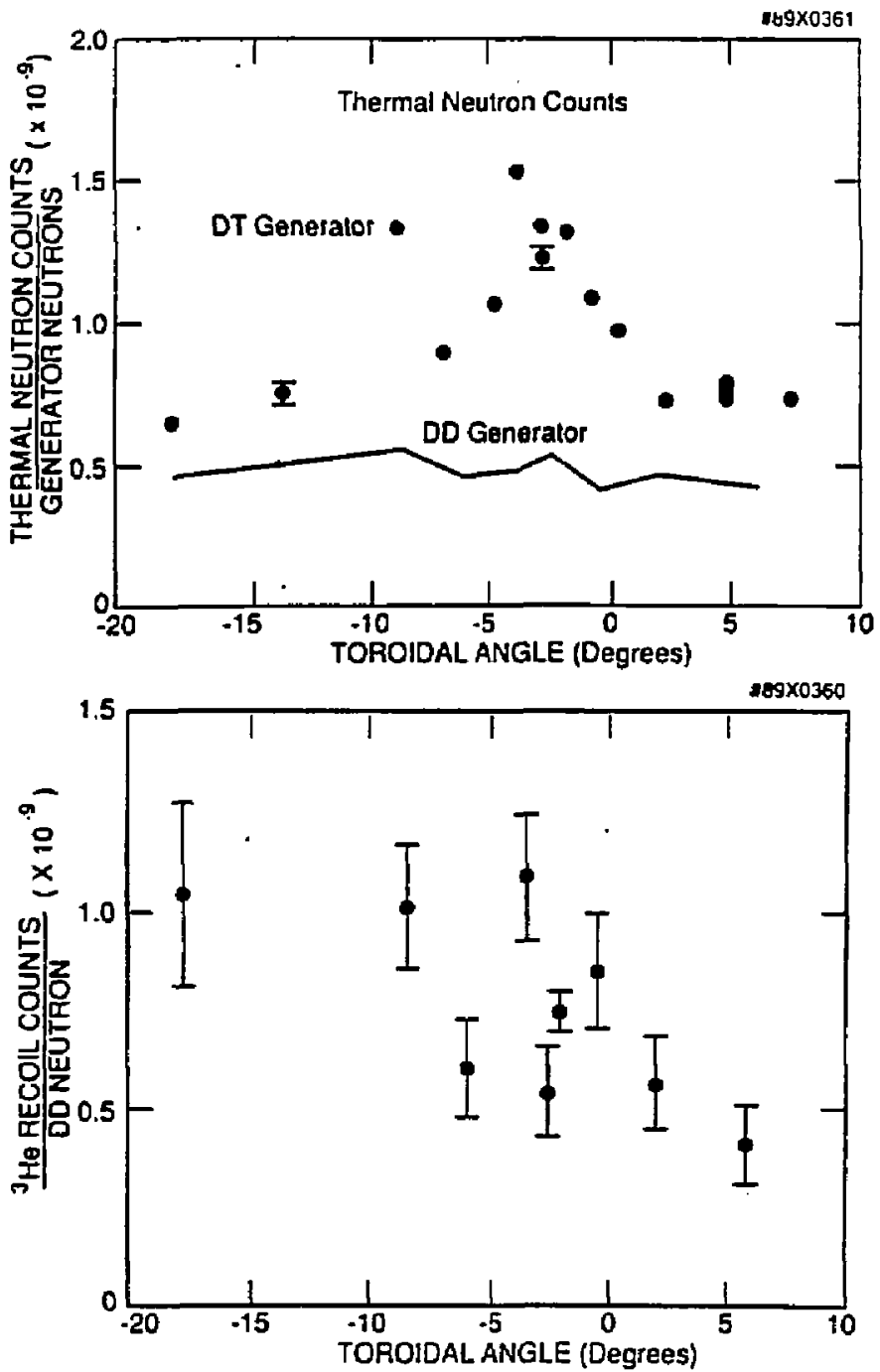

Figure 19: ${ }^{3} \mathrm{He}$ aperture maps. (Top) The count rate of thermal neutron events for the DT and DD generator scans in Bay F. (Bottom) The ${ }^{3} \mathrm{He}$ recoil coūnts for the Bay $F$ scan of the $\mathrm{DD}$ generator. 
Table 5: Efficiency of the ${ }^{3} \mathrm{He}$ detector observed during the $3 \mathrm{hr}$ generator run (total directed yield of $2.32 \times 10^{12}$ neutrons) compared to that expected for the response function.

\begin{tabular}{l|cc} 
& \multicolumn{2}{|c}{ Point Source Efficiencies } \\
& 3 Hr Exp. & Response Function \\
\hline Thermals & $7.8 \times 10^{-10} \pm 12 \%$ & 0 \\
3He Recoils & $4.0 \times 10^{-11} \pm 32 \%$ & $2.7 \times 10^{-10} \pm 25 \%$ \\
DD Peak & $7.8 \times 10^{-12} \pm 70 \%$ & $8.5 \times 10^{-11} \pm 25 \%$
\end{tabular}

\subsubsection{Detector efficiency}

The intrinsic efficiency of the $2.5-\mathrm{MeV}$ neutron peak [18] is about $6 \times 10^{-5}$. (This value is taken from Figure 9 of Ref. IB, and disagrees from the values in Table 3 of that reference by a factor of 10 .) The recoil edge efficiency is obtained by comparing the ratio of the number of counts in the response function under the peak and under the recoil edge over the $500 \mathrm{keV}$ ( 60 channels) iegion that is summed. These intrinsic efficiencies can be multiplied by the geometrical factor for the spectrometer to obtain point source efficiencies. The active area of the detector is $5 \mathrm{~cm} \times 15 \mathrm{~cm}$ and about $1 / 2$ of the detector has a direct view of the generator. For the detector $14.7 \mathrm{~m}$ from the torus the geometrical factor $A / 4 \pi L^{2}$ is $1.4 \times 10^{-8}$. The observed point source efficiencies from the 3 -hour generator exposure for the ${ }^{3} \mathrm{He}$ ionization chamber are about an order of magnitude lower than expected for these types of detectors (Table 5). Other efficiency measurements[21,22] for this type of detector have found efficiencies similar in magnitude to the detector response function. Presumably, the reduced efficiency is due to absorption and scattering by the material between the generator and the detector (see Table 3). From the removal cross sections[19] for these materials, we expect the $2.5-\mathrm{MeV}$ neutron flux to be attenuated by a factor-of14. The ratio of the DD peak response and the ${ }^{3} \mathrm{He}$ recoils is in agreement (within statistical uncertainties) with the response function again indicating that the ${ }^{3} \mathrm{He}$ recoil edge can be potentially useful for establishing the absolute detector efficiency, since it has more counts and, therefore, lower statistical uncertainties and, as well, a lower background (Fig. 12). A complication is that the pile-up of the thermal neutron peak with itself occurs near the ${ }^{3} \mathrm{He}$ recoil edge. The addition of a pile-up rejection circuit to the detector electronics would improve the utility of the ${ }^{3} \mathrm{He}$ recoil edge since it is desirable to operate the detector at maximum count rates in order to accumulate full energy counts. 
Table 6: ${ }^{3} \mathrm{He}$ Detector Efficiency (counts per neutron) for Plasma Neutrons

\begin{tabular}{l|ccc} 
& $3 \mathrm{hr}$ Exp. & Shot 30255 & Bay F Scan \\
Equivalent & & & \\
Plasma yjeld & $\frac{300}{15} \times 2.32 \times 10^{12}$ & $4.01 \times 10^{13}$ & $\frac{300}{10} \times 1.02 \times 10^{12}$ \\
\hline Thermals & $3.9 \times 10^{-11} \pm 25 \%$ & $7.9 \times 10^{-11} \pm 20 \%$ & $4.4 \times 10^{-11}=12 \%$ \\
${ }^{3} \mathrm{He}$ Recoils & $2.0 \times 10^{-12} \pm 40 \%$ & $2.9 \times 10^{-12} \pm 29 \%$ & $1.3 \times 10^{-12} \pm 78 \%$ \\
$2.5-\mathrm{MeV}$ n's & $3.9 \times 10^{-13} \pm 72 \%$ & $4.5 \times 10^{-13} \pm 42 \%$ & $<4.9 \times 10^{-13}$
\end{tabular}

\subsubsection{Comparison to plasma yields}

The efficiency of the ${ }^{3} \mathrm{He}$ ionization chamber for TFTR plasma neuirons was obtained using shot 30255 where the neutron emission[2] was measured using the epithermal neutron system with its own calibration. A measurable DD 2.5-MeV neutron peak and ${ }^{3} \mathrm{He}$ recoil proton spectrum were obtained on this single plasma shot which had a total neutron emission from the ${ }^{235} \mathrm{U}$ detector of $(4.0 \pm 1.0) \times 10^{13}$ neutrons.

The efficiency of the ${ }^{3} \mathrm{He}$ ionization chamber for $2.5-\mathrm{MeV}$ neutrons inside the TFTR vacuum vessel was obtained from the $3 \mathrm{hr}$ run of the DD generator. In this case, ihe generator can approximate the plasma by using the measured collimator aperture (Fig. 18). The equiralent isotropic emission is the directed yield divided by the fraction of the torus seen by the collimator. That fraction is about $18 / 360$ where the $18^{\circ} \pm 4^{\circ}$ represents either the measured collimator aperture $\left(I / \epsilon_{\mathrm{DD}}\left(2^{\circ}\right)\right)$ or one Bay. Unfortunately, the background subtraction significantly interfered with the accuracy of this calibration and resulted in the large quoted uncertainty (Table 6). Still, the efficiencies agree with the plasma case.

Another method of calculating the efficiency of the ${ }^{3} \mathrm{He}$ ionization chamber to TFTR 2.5-MeV neutrons is to use the Bay F scan. Taking a set of 10 minute irradiations (shots 90017,90020,90021, and 90023-90028) that are nearly evenly spaced throughout Bay $F$, the aperture does not need to be known since the equivalent isot ropic emission at the perpendicular rate from the full torus is obtained by multiplying the directed yield from these shots by the number of Bays. Although the 2.5-MeV peak could not be resolved, the efficiencies for the ${ }^{3} \mathrm{He}$ recoil edge and the thermal peak were the same as for the 3 hr exposure (Table 6). 


\section{Conclusions}

The absolute efficiency of collimated NE213 and ${ }^{3} \mathrm{He}$ neutron spectrometers has been determined experimentally using $2.5-\mathrm{MeV}$ and $14-\mathrm{MeV}$ neutrons produced in neutron generators that were placed inside the TFTR vacuum vessel.

The NE213 efficiency for 14-MeV reutrons is within $30 \%$ of that expected from previous triton bumup studies[5] where the signals had been cross calibrated to the fission detectors $[9,8$. It agrees within the $20 \%$ relative calibration error of the DT calibration of the ${ }^{235} \mathrm{U}$ detector $[9]$, if insignificant $\mathrm{DD}$ neutrons are present late in time[23]. The NE213 DT efficiency would be 1.5 times too low if there were 1/2 as many DD as DT neutrons, a value consistent with the ${ }^{234} U$ DT calibration[8]. The NE213 efficiency for $2.5-\mathrm{MeV}$ neutrons agrees with the fission detectors within the $25 \%$ relative calibration error for both ohmic and neutral beam injected discharges, and over a small range of major radius. The agreement is good despite the lack of a two-dimensional map of the view of the tokamak and it's varying attenuation. The absolute calibration for the magnitude of the triton burnup is known within $20 \%$, . where the small uncertainty occurs since several systematic errors can be eliminated from the ratio once both fluxes were measured by using the same delector.

The accuracy of the ${ }^{3} \mathrm{He}$ detector calibration was limited due to a background (approximately $10^{-1}$ counts per minute) in the $2.5-\mathrm{MeV}$ neutron energy range. The background is probably caused by microarcing. Nevertheless, the detestor response to 2.5-MeV neutrons cither from the $d(n, p) t$ reactions or the ${ }^{3} \mathrm{He}$ recoil events is within about $50 \%$ of that obtained from neutrons from TFTR plasmas. This means that the ${ }^{3}$ He detector calibration was consistent with the calibration of the epithermal neut ron detectors. A striking feature of the observed spectra is that the neutron spectrum above about $2 \mathrm{MeV}$ is composed primarily of full energy neutrons. This result means that a recoil spectrometer such as the NE213 scintillator can usefully use a 2-Mel threshold to eliminate most of the scattered neutrons from its detector response.

The effects of attenuation on the detector efficiencies is significant. From the estimated amount of material between the plasma and the spectrometers, the DD neistron attenuation was measured to be about 3 to 4 times that expected for the NE213 detector, and approximately equal to about half that expected for the ${ }^{3} \mathrm{He}$ ionization chamber. Considering the wide variation in material thickness in the collimat! i field of view, uncertainties in the absolute detector counting efficiencies, and the differing energy thresholds of the spectrometers, this is considered to be reasonable agreement of the experimentally measured efficiencies and the theoretical expectations. 


\section{Acknowledgments}

The authors thank Steve Bosch, Manfred Frey, Hans Hendel, Dan Jassby, Larry Johnson, Harold Murphy, Tom Murphy, Ernie Nieschmidt, Janet Roberts, Tet Saito, Graeme Tait, Bob Waszuzak, and Ken Young for help in the calibration. We thank Fans Hendel and Dan Jassby for providing fission chamber results for comparing neutron rates. We also acknowledge Stewart Zweben for providing unpublished results from the $1985{ }^{262} \mathrm{Cr}$ calibration. Janet Felt wrote the computer code that aided in the preparation of Fig. 15.

This work was supported by the U.S. Department of Energy Contract No. DEAC02-76-CHO-3073. 


\section{References}

[1] J. V. Foley, TFTR Ion Temperature Measurements by High Resolution Neutron Spectroscopy, Master's thesis, Princeton University, 1985, (Department of Chemical Engineering).

[2] T. Nishitani and J. D. Strachan, Neutron Spectroscopy on TFTR, Technical Report PPPL-2512, Princeton Plasma Physies Laboratory, Princeton, NJ, May 1988.

[3] J. D. Strachan, T. Nishitani, and C. W. Barnes, Neutron Spectroscopy on TFTR. Rev. Sci. Instrum. 59(8), 1732-1734 (1988).

i4] W. R. Burrus and V. V. Verbinski, Fast-Neutron Spectroscopy with Thick Organic Scintillators, Nucl. Instrum. Methods 67, 181-196 (1969).

[5] C. W. Barnes, H.-S. Bosch, E. B. Nieschmidt, T. Saito, M. Bitter, et al., Triton Burnup Studies on TFTR, in Proc. of the 15th European Conference on

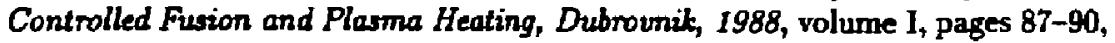
Petit-Lancy, 1988, EPS.

[6] E. B. Nieschmidt, T. Saito, C. W. Barnes, H.-S. Bosch, and T. J. Murphy, Calibration of the TFTR Neutron Activation System, Rev. Sci. Instrum. 59(8), 1715-1717 (1988).

[7] H.-S. Bosch, J. D. Strachan, C. W. Barnes, and E. B. Nieschmidt, Calibration of a Surface Barrier Detector for 14-MeV Neutron Flux Measurements on TFTR. Rev. Sci. Instrum. 59(8), 1718-1720 (1988).

[8] D. L. Jassby, H. W. Hendel, and H.-S. Bosch, Relative Intensities of 2.5- and 14-MeV Source Neutron from Comparative Responses of U-235 and U-238 Detectors, Rev. Sci. Instrum. 59(8), 1688-1690 (1988).

[9] H. W. Hendel, D. L. Jassby, H. S. Bosch, C. W. Barnes, L. C. Johnson, T. J. Murphy, E. B. Nieschmidt, T. Saito, J. D. Strachan, G. D. Tait, and K. M. Young, TFTR Epithermal Neutron Detector System: Recalibration and Effect of Nonisotropic Neutron Emission, Rev. Sci. Instrum. 58(8), 1682-1684 (1988).

[10] E. B. Nieschmidt, A. C. England, H. W. Hendel, D. L. Hillis, J. A. Isaacson, L. P. $\mathrm{Ku}$, and F. Y. Tsang, Effects of Neutron Energy Spectrum on the Efficiency Calibration of Epithermal Neutron Detectors, Rev. Sci. Instrum. 56(5), 10841086 (1985). 
11] J. D. Strachan. P. Colestock, H. Eubank. I. Grisham, J. Hovey, G. Schilling, L. Stewart, W. Stodiek, R. Stooksberry, and K. M1. Young, Mleasurement of the Neutron Spectra from Beam-Heated PLT Plasmas, Nature 279(5714), 626-628 (197!).

12] R. E. Chrien, Measurements of Furion Reactions from a Tokamak Plasma, PhD thesis, Princeton University, Oct. 1981.

13] V. V. Verbinski, W. R. Burrus, T. A. Love, W. Zobel, and N. W. Hill, Calibration of an Organic Scintillator for Neutron Spectrometry, Nucl. Instrum. Methods 65, 8-25 (1968).

14] H. H. Knox and T. G. Miller, A Technique for Determining Bias Settings for Organic Scintillators, Nucl. Instrum. Methods 101, 519-525 (1972).

15] M. Drosg, Accurate Measurement of the Counting Efficiency of a NE-213 Neutron Detector between 2 and $26 \mathrm{MeV}$, Yucl. Instrum. Methods 105, 573-584 (1972).

16] L. J. Perkins and M. C. Scott, The Application of Pulse Shape Discrimination in NE 213 to Neutron Spectrometry, Nucl. Instrum. Methods 166, 451-464 (1979).

17] J. K. Dickens, J. W. McConnel, K. M. Chase, H. W. Hendel, E. B. Nieschmidt, and F. Y. Tsang, Measurements of the Neutron and gamma-Ray Fluences in the TFTR Test Cell due to a Point Source Simulating D-T Fusion Plarma Neutron Production, Fusion Technology 12, 270-280 (1987).

18) K. Beimet, G. Nyman, and $O$. Tengblad, Response Function for ${ }^{3} \mathrm{He}$ Neutron Spectrometers, Nucl. Instrum. Methods Phys. Research A245, 402-414 (1386).

19] A. B. Chilton, J. K. Shultis, and R. E. Law, Principles of Radiation Shielding, Prentis-Hall, 1984, (see Section 8.3).

20] T. J. Murphy, Private communication (February 2, 1988), based on the data taken by L. Samuelson.

21] W. A. Fisher, S. H. Chen, D. Gwinn, and R. R. Parker, A Fast Nẹutron Spectrometer for D-D Fusion Neutron Measurements at the Alcator $C$ Tokamak, Vucl. Instrum. Methods Phys. Research 219, 179-191 (1984).

22! O. N. Jarvis, G. Gorini, M. Hone, J. Källne, G. Sadler, V. Merlo, and P. van Belle, Neutron Spectrometry at JET, Rev. Sci. Instrum. 57(8), 1717-1722 (1986). 
[23] D. L. Jassby, H. W. Hendel, C. W. Barnes, S. Bosch, F. E. Cecil, D. C. McCune, E. B. Nieschmidt, and J. D. Strachan, Fission-Detector Determination of D-D Triton Burnup Fraction in Beam-Heated TFTR Plasmas, in Proc. of the 14th European Conference on Controlled Fusion and Plasma Physics, Madrid, 1987, volume III, pages 1264-1267, Petit-Lancy, 1987, EPS. 